\title{
Polymerase Theta Inhibition Kills Homologous Recombination Deficient Tumors
}

Jia Zhou', Camille Gelot ${ }^{2}$, Constantia Pantelidou ${ }^{3}$, Adam Li', Hatice Yücel², Rachel E. Davis $^{4}$, Anniina Farkkila1 ${ }^{1}$, Bose Kochupurakkal ${ }^{1}$, Aleem Syed ${ }^{5}$, Geoffrey I. Shapiro ${ }^{3,6}$, John A. Tainer ${ }^{5}$, Brian S. J. Blagg ${ }^{4}$, Raphael Ceccaldi ${ }^{2,7^{*}}$ and Alan D. D'Andrea ${ }^{1,6,7^{*}}$

${ }^{1}$ Department of Radiation Oncology, Dana-Farber Cancer Institute, Harvard Medical School, Boston, MA 02215, USA.

${ }^{2}$ Inserm U830, PSL Research University, Institut Curie, 75005, Paris, France.

${ }^{3}$ Department of Medical Oncology, Dana-Farber Cancer Institute and Department of Medicine, Harvard Medical School, Boston, Massachusetts, USA.

${ }^{4}$ Department of Chemistry and Biochemistry, University of Notre Dame, Notre Dame, IN 46556, USA.

${ }^{5}$ Departments of Cancer Biology and of Molecular and Cellular Oncology, University of Texas MD Anderson Cancer Center, Houston, TX 77030, USA.

${ }^{6}$ Center for DNA Damage and Repair, Dana-Farber Cancer Institute, Boston, MA, USA.

${ }^{7}$ Co-senior authors.

* Co-corresponding authors.

\section{* Corresponding authors:}

Alan D. D'Andrea, M.D.

Director, Susan F. Smith Center for Women's Cancers (SFSCWC)

Director, Center for DNA Damage and Repair

Dana-Farber Cancer Institute

The Fuller-American Cancer Society Professor

Harvard Medical School

Phone: 617-632-2080

Email: Alan_Dandrea@dfci.harvard.edu

Raphael Ceccaldi

Institut Curie, 75005, Paris, France

Phone: +33 (0)156246949

Email: raphael.ceccaldi@curie.fr

Key Words: Novobiocin, Polymerase theta (POL $\theta)$, Homologous Recombination, PARP inhibitor resistance. 


\section{Abstract}

PARP inhibitors (PARPi) have become a new line of therapy for Homologous Recombination (HR)-deficient cancers. However, resistance to PARPi has emerged as a major clinical problem. DNA polymerase theta $(P O L \theta)$ is synthetic lethal with $\mathrm{HR}$ and a druggable target in HR-deficient cancers. Here, we identified the antibiotic Novobiocin (NVB) as a specific POL $\theta$ inhibitor that selectively kills HR-deficient tumor cells in vitro and in vivo. NVB directly binds to the POLO ATPase domain, inhibits its ATPase activity, and phenocopies POL $\theta$ depletion. BRCA-deficient tumor cells and those with acquired PARPi resistance are sensitive to NVB in vitro and in vivo. Increased POL $\theta$ expression levels predict NVB sensitivity. The mechanism of NVB-mediated cell death in PARPi resistant cells is the accumulation of toxic RAD51 foci, which also provides a pharmacodynamic biomarker for NVB response. Our results demonstrate that NVB may be useful alone or in combination with PARPi in treating HR-deficient tumors, including those with acquired PARPi resistance.

One Sentence Summary: We identified Novobiocin as a specific POL $\theta$ inhibitor that selectively kills naïve and PARPi resistance HR-deficient tumors in vitro and in vivo. 
Since the discovery of the synthetic lethal relationship between PARP inhibition and homologous recombination $(\mathrm{HR})$ repair deficiency $(1,2)$, PARP inhibitors (PARPi) have become a new line of therapy for HR-deficient cancers (3-7). However, resistance to PARPi has emerged as a major obstacle to their clinical effectiveness (8). While several mechanisms of PARPi resistance are known (9-14), a rational and effective method for overcoming such resistance is still lacking. DNA polymerase theta (POL $\theta$ or POLQ), a key enzyme in microhomology-mediated end joining (MMEJ), is synthetic lethal with HR and a druggable target in HR-deficient cancers (15-18). A small-molecule screen identified novobiocin (NVB) as a specific POLQ inhibitor. NVB inhibited POLQ in vitro and in vivo, Moreover, we demonstrated that NVB can be used alone or in combination with PARPi in treating HR-deficient tumors, even after they have acquired PARPi resistance.

\section{RESULTS}

\section{A small-molecule screen identified NVB as a specific POL $\theta$ inhibitor}

Polymerase theta (POL $\theta$, encoded by the POLQ gene) is a critical enzyme for repairing DNA double-strand breaks (DSBs) through a microhomology-mediated end joining (MMEJ) mechanism $(19,20)$. POL $\theta$ expression is elevated in several types of cancers where it correlates with poor prognosis $(21,22)$. HR-deficient tumors upregulate POL $\theta$ mediated MMEJ as a survival strategy, and loss of POL $\theta$ in these tumors results in cell death $(15,17)$. This synthetic lethality with $\mathrm{HR}$ defines the rationale for developing POL $\theta$ inhibitors for the treatment of HR-deficient cancers (16).

The ATPase activity of POL $\theta$ is required for the survival of HR-deficient cells (17). We took advantage of the strong in vitro ATPase activity of purified POLO (Fig. S1A) and designed a large-scale small-molecule screen to identify POL $\theta$ inhibitors based on the ADPGlo luminescent assay (Fig. 1A, B). The hits from the primary screen were evaluated in a 
secondary screen (Fig. S1B-D). The four most potent POL $\theta$ ATPase inhibitors were Mitoxantrone (MTX), Suramin (SUR), Novobiocin (NVB) and Aurintricarboxylic acid (AUR), which were individually verified in a ${ }^{32} \mathrm{P}$-based radiometric ATPase assay (Fig. S1E). To test the specificity of the most potent compounds, we purified two other known DNA repairrelated ATPases, namely SMARCAL1 and $\operatorname{CHD1}(23,24)$, and performed ATPase inhibition assays. Only NVB showed high specific inhibition of POL $\theta$ with little effect on SMARCAL1 or CHD1 activity, while MTX, SUR and AUR were nonspecific pan-ATPase inhibitors that inhibited all three ATPases (Fig. 1C and S1F, G).

To further evaluate the specificity of Novobiocin (NVB) for POL $\theta$, we analyzed its binding capacity. NVB-conjugated beads pulled down the purified ATPase domain of POL $\theta$ but not of SMARCAL1, suggesting that NVB directly binds to the POL $\theta$ ATPase domain in vitro (Fig. 1D, S1H). Active NVB-beads also pulled down GFP-tagged full-length POL $\theta$ overexpressed in HEK293T cells (Fig. S1I). Excess free NVB competed the POL $\theta$ binding to the NVB-beads (Fig. S1J). Next, we performed protein thermal shift assays, an assay commonly used to demonstrate ligand-protein binding $(25,26)$. NVB significantly increased the thermal stability of POL $\theta$ when compared to DMSO (Fig. 1E), while the PARP1 inhibitor olaparib, used as an internal control, effectively stabilized the PARP1 protein (Fig. S1K). Finally, we performed molecular docking to find the NVB binding site using the published crystal structure of POLQ ATPase domain (27). The computational docking results revealed that a deep tunnel within the POLQ ATPase domain could accommodate NVB, and that one end of NVB could reach the active site to prevent ATP binding to POLQ and inhibit its ATPase activity (Fig. 1F). Taken together, these data demonstrate that NVB binds directly and specifically to the POLO ATPase domain in vitro.

We next assessed whether cellular exposure to NVB has the same consequences as POLO depletion. Both the ATPase and the polymerase domains contribute to efficient 
MMEJ repair activity (28), and the PARP-dependent recruitment of POL $\theta$ to DSB sites is a crucial step in this function $(15,17)$. The inhibition of POL $\theta$ by NVB prevented the recruitment of POL $\theta$ to laser micro-irradiated DNA damage sites in human cells (Fig. 1G). Consequently, NVB inhibited the MMEJ activity in U2OS cells, as demonstrated by the GFPbased EJ2 repair assay (Fig. $\mathbf{1 H})$. In contrast, NVB had little effect on HR activity, as measured by the DR-GFP reporter assay (Fig. S2A). POL $\theta$ also functions as an antirecombinase that antagonizes $\mathrm{HR}$, and depletion of $\mathrm{POL} \theta$ increases $\mathrm{RAD51}$ (17) and $\mathrm{VH} 2 \mathrm{AX}(29,30)$ foci assembly. Again, cellular exposure to NVB recapitulated the RAD51 and $\mathrm{yH} 2 \mathrm{AX}$ phenotypes of a POL $\theta$ knockdown. NVB activated more RAD51 foci (Fig. S2B) and $\mathrm{yH} 2 \mathrm{AX}$ foci (Fig. S2C) in RPE1 cells, regardless of IR exposure. Taken together, these results establish that NVB-mediated POL $\theta$ inhibition impairs POL $\theta$ DNA repair function and phenocopies POL $\theta$ depletion in human cells.

\section{NVB specifically Kills HR-deficient cells in vivo and in vitro}

Depletion of POL $\theta$ is synthetically lethal in HR-deficient tumor cells $(15,17)$. Since NVB has been previously approved for human use, we decided to directly test whether NVB reduces the survival of HR-deficient tumor cells in animal models. First, we evaluated the efficacy of NVB in tumors derived from the K14-Cre-Brca1 ${ }^{f / f} ; T_{r p 53^{f / f}}$ genetically-engineered mouse model (GEMM) of triple-negative breast cancer (TNBC), in which spontaneous mammary carcinomas develop after approximately 7 months $(31,32)$. Tumors from this model were transplanted into immunocompetent FVB/129P2 syngeneic mice that were subsequently treated with NVB. NVB strongly suppressed the growth of these Brca1deficient GEMM-derived tumors (Fig. 2A and individual mouse data in Fig. S3A). The NVBtreated tumors were significantly smaller compared to the vehicle-treated after 7 days of treatment and beyond. NVB prolonged the overall survival of the tumor-bearing mice by 3- 
fold compared to vehicle, with median survival times of 29 and 10 days for NVB- and vehicletreated mice, respectively (Fig. 2B).

Next we tested whether the efficacy of NVB in vivo was specific to HR-deficient tumors. We performed a mouse xenograft study with a FANCF-deficient, HR-deficient ovarian cancer cell line [TOV21G + empty vector (EV)] or with the FANCF-complemented, HR-proficient cell line (TOV21G + FANCF cDNA) (Fig. 2C). TOV21G + EV cells but not TOV21G + FANCF cDNA cells were sensitive to olaparib and NVB (Fig. S3B, C) (33). While vehicle treatment had no impact on the growth of FANCF-deficient or FANCFcomplemented tumors, NVB specifically impaired the growth of the FANCF-deficient tumors, with no effect on FANCF-complemented tumors (Fig. 2C). We also measured RAD51 foci as a pharmacodynamic biomarker and showed that RAD51 foci were strongly induced in the treated tumors (Fig. S3D-F). These results demonstrate that NVB strongly and specifically suppresses the growth of HR-deficient tumors in vivo.

To confirm and strengthen the in vivo results, we studied isogenic pairs of HRdeficient and HR-proficient cells in vitro. We generated BRCA1 (34) and BRCA2 knockout RPE1 cells (referred as $B R C A 1^{-/-}$and $B R C A 2^{-/}$) in a $T P 53^{-/-}$background, which were sensitive to PARPi (Fig. S4A). Consistent with our in vivo data, clonogenic survival assays showed that NVB significantly reduced the survival of $B R C A 1^{-/}$and $B R C A 2^{-/}$cells, compared to the isogenic WT cells (Fig. 2D, S4B and Fig. S4C). We also tested the top 15 initial hits from our screen for their ability to differentially affect the survival of $B R C A 1^{--}$and WT cells. Among those, NVB had the highest efficacy in selectively killing the $B R C A 1^{\%}$ cells (Fig. S4D).

To demonstrate the cellular outcome of NVB exposure, we next showed that NVB exposure induces apoptosis in $B R C A 1^{-/-}$but not WT cells in a dose dependent manner (Fig. S5A). NVB induced chromosomal aberrations and radial chromosomes (a marker of 
genomic instability) in $B R C A 1^{-}$cells, even in the absence of the crosslinking agent mitomycin C (MMC) (Fig. S5B-D), confirming that NVB increases DNA damage. These results further demonstrate that NVB phenocopies POL $\theta$ depletion, and indicate that NVBmediated POLO inhibition is synthetically lethal with HR deficiency and specifically induces cell death in HR-deficient tumor cells.

\section{POL $\theta$ is the major target of NVB in human cells}

Off target effects are major concerns of biological small inhibitors. To further evaluate whether POLO is the specific target of NVB and to demonstrate the specificity of NVB in cells, we knocked out the POLQ gene in U2OS and RPE1 cells using CRISPR-Cas9 and asked whether loss of POL $\theta$ alleviates the cytotoxicity of NVB (Fig. 2E and S6). POLQ knockout cells were confirmed by genomic sequencing, immunoblotting, increased RAD51 foci, increased sensitivity to irradiation, and increased micronuclei formation (Fig. S6A-F). Importantly, $P O L Q^{-/}$cells showed increased tolerance to NVB, both in U2OS and RPE1 cells, as compared to the WT cells (Fig. 2E and S6G), indicating that POL $\theta$ is the major target of NVB in human cells.

NVB is a known coumarin antibiotic that inhibits DNA gyrase in bacteria (35). NVB has been reported to inhibit the ATPase of HSP90 and of the DNA gyrase homolog topoisomerase II (TOP2) in eukaryotes (36) (37) (38), suggesting that these enzymes may be off-target enzymes of NVB. However, other reports suggest that NVB does not inhibit TOP2 in eukaryotic cells (39). Additionally, the reported IC 50 of these targets are very high (approximately $700 \mu \mathrm{M}$ for HSP90 and $300 \mu \mathrm{M}$ for TOP2), compared with the IC50 of POL $\theta$. Nevertheless, we next examined whether the cytotoxic effect of NVB in HR-deficient cells resulted from off-target inhibition of HSP90 or TOP2. Unlike the well-characterized HSP90 inhibitor PU-H71, NVB treatment at a concentration that killed HR-deficient cells (i.e. 100 
$\mu \mathrm{M})$ did not induce the degradation of HSP90 clients such as AKT1, BRCA1, or CDK6, or increase the expression of HSP70 (Fig. S7A-C). These results suggest that the cytotoxic effect of NVB is not related to HSP90 inhibition. Furthermore, the combination of NVB and the TOP2 inhibitor etoposide exhibited additive cytotoxicity in TOV21G and CAPAN1 cells (Fig. S7D, E), suggesting that NVB and etoposide do not share TOP2 as a target and that NVB cytotoxicity is unrelated to TOP2 inhibition.

\section{NVB potentiates the effect of PARPi in HR-deficient tumors}

PARPi are currently being evaluated in different combination therapy settings with cytotoxic chemotherapy, radiation, targeted therapies, and immunotherapies (40). We explored whether the combination of NVB and a PARPi may be more effective than PARP inhibition alone in killing HR-deficient tumors. Indeed, NVB further sensitized $B R C A 1^{-/-}$ RPE1 cells to the PARPi rucaparib, but not BRCA-proficient WT cells in clonogenic assays

(Fig. 2F). We studied the combination with a second PARPi, olaparib, where synergism of NVB and olaparib was also observed in HR-deficient TOV21G+EV cells, but not in HRproficient TOV21G+FANCF cells (Fig. S7F). Importantly, NVB reduced the IC 50 of rucaparib by more than 20 -fold in $B R C A 1^{-/-}$RPE1 cells and the $\mathrm{IC}_{50}$ of olaparib more than 40 -fold in TOV21G cells compared to their respective HR-proficient counterparts (Fig. 2F and S7F). One strategy to prevent acquired resistance during treatment is combination therapy. The observed synergy between NVB and PARPi prompted us to evaluate whether NVB could overcome PARPi resistance in HR-deficient tumors.

\section{POL $\theta$ inhibition by NVB overcomes acquired PARPi resistance in vivo and in vitro}

Multiple PARP inhibitors (PARPi) including olaparib, niraparib, rucaparib, and talazoparib have received approval for the treatment of ovarian and breast tumors with HR 
bioRxiv preprint doi: https://doi.org/10.1101/2020.05.23.111658; this version posted May 26, 2020. The copyright holder for this preprint (which was not certified by peer review) is the author/funder, who has granted bioRxiv a license to display the preprint in perpetuity. It is made available under ACC-BY-NC-ND 4.0 International license.

deficiency (41-43). Despite the remarkable initial tumor response and improved progression-free survival (PFS) in HR-deficient patients (4-6, 44), acquired PARPi resistance is emerging as an unmet medical need (8).

To evaluate whether $\operatorname{POL} \theta$ inhibition could represent a novel therapeutic option for PARPi-resistant tumors, we first tested the efficacy in vivo of NVB in the patient-derived xenograft (PDX) model DF83, generated from an HR-deficient (loss of RAD51C expression), human high-grade serous ovarian carcinoma (45). NSG mice bearing the HRdeficient DF83 ovarian cancer PDX model were treated with olaparib, NVB, or a combination of the two, and tumor growth was monitored by bioluminescence imaging (BLI) (Fig. 3A). In order to determine the combined effect with NVB, olaparib was delivered at a submaximal dose (50 mg/kg daily), and we observed only a small degree of tumor growth inhibition. Treatment of DF83 with NVB alone (75 mg/kg, twice a day) led to tumor regression, while tumors in vehicle-treated mice showed exponential growth (Fig. 3B). Strikingly, when the DF83 tumor-bearing mice were given NVB and olaparib in combination, we observed complete tumor regression, and few tumor cells were detectable via BLI on day 28 in this group (Fig. 3A-B). These in vivo results suggest NVB may be useful in combination with PARPi to achieve better efficacy and prevent drug resistance in HR-deficient tumors. No significant toxicity to normal mouse tissue was observed from the drug combination.

Next, we evaluated the efficacy of NVB alone and in combination with olaparib in the PARPi-resistant PDX model DF59, which was isolated from a patient with a germline BRCA1 mutation and acquired PARPi resistance. This model harbors biallelic mutations in the TP53BP1 locus, providing a mechanism of the acquired PARPi resistance (45). In the DF59 PDX model, there was no response to olaparib monotherapy, but NVB monotherapy substantially reduced tumor growth, indicating a lack of cross-resistance between PARP inhibition and NVB. Importantly, the combination of NVB and olaparib further inhibited DF59 
tumor growth, with tumor regression in the first two weeks (Fig. 3C-D). In contrast to DF83 and DF59 models, the BRCA1 WT, HR proficient PDX model DF149 was resistant to both drugs and to the combination (Fig. 3E-F). These data demonstrate that NVB can inhibit the growth of a BRCA1-mutated, PARPi-resistant tumors in vivo and that the combination of NVB and a PARPi is particularly effective in treating at least some PARPi-resistant tumors.

\section{RAD51 focus is a pharmacodynamic biomarker for NVB response in vivo}

We previously showed that depletion of POL $\theta$ in HR-deficient tumors resulted in RAD51 accumulation (17). Similarly, 53BP1 and POL $\theta$ are synthetic lethal, and the double knockout cells accumulate large, toxic RAD51 foci (46). We thus reasoned that NVB may induce RAD51 foci in HR-deficient tumors with PARPi resistance in vivo, and the RAD51 foci may serve as a pharmacodynamic biomarker for NVB response. To test this hypothesis, we collected tumor cells from the PARPi and/or NVB treated PDX models and evaluated RAD51 foci by immunohistochemistry (Fig. 3G). Since DF83 is an HR-deficient and PARPi sensitive model, RAD51 foci were not observed. The HR-restored and PARPi-resistant DF59 model, with biallelic 53BP1 mutations, exhibited a strong increase in RAD51 foci when treated with NVB alone or in combination with olaparib (Fig. 3G). Similarly, TOV21G xenograft tumors also exhibited increased RAD51 foci by immunohistochemistry in vivo after NVB treatment, which was further enhanced by olaparib (Fig. S3D-F). Results from these two in vivo studies demonstrate that RAD51 focus can serve as a pharmacodynamic biomarker for the response of tumors to NVB or other POL $\theta$ inhibitors. Interestingly, we also observed that two NVB-responsive tumor models had elevated levels of POL $\theta$ mRNA expression, compared to the NVB-resistant DF149 model, suggesting that elevated POL $\theta$ may be a useful predictive biomarker for POL $\theta$ inhibitor response (Fig. 3H). 


\section{NVB overcomes multiple PARPi resistance mechanisms but not perfect BRCA}

\section{reversions}

To better understand the mechanism by which NVB overcomes PARPi resistance and, we generated several PARPi-resistant clones from $B R C A 1^{-1} R P E 1$ cells by gradually exposing them to increasing concentrations of PARPi (Fig. 4A). We selected four clones (named R1 to R4), all of which have acquired resistance to olaparib (Fig. S8A-C). Multiple PARPi resistance mechanisms were identified in these clones, corresponding to published mechanisms (8). Replication fork stabilization was evident in the R1 and R2 clones (Fig. S8D). The R1, R3, and R4 clones have restored RAD51 foci, a marker for restored HR activity (Fig. S8E). The restoration of HR repair in these clones may have resulted in part from their downregulation of NHEJ repair. Indeed, the R3 clone had decreased REV7 expression, and the R4 clone had decreased 53BP1 expression (Fig.S8F-G). None of the clones had re-expressed BRCA1, thereby excluding somatic reversion of BRCA1 as an underlying PARPi resistance mechanism (Fig. 4B and S8H). Strikingly, all four PARPiresistant clones remained sensitive to NVB to a similar extent as the parental RPE-BRCA1/- cells (Fig. 4C-D), suggesting that NVB may overcome multiple mechanisms of acquired resistance to PARPi. To confirm that POL $\theta$ inhibition was responsible for cell death in the PARPi-resistant $B R C A 1^{-/-}$clones treated with NVB, we knocked out POLQ in R1, R2, and the parental cells and measured their cell survival. Upon POLQ knockout, the PARPiresistant clones showed reduced survival similar to the parental $B R C A 1^{-/-}$cells, demonstrating that POLQ inhibition was the mechanism for NVB cytotoxicity in these cells (Fig. S8I).

Next, we examined more clinically relevant PARPi-resistant, HR-deficient models, using tumor cells derived from patients. First, we evaluated the effect of NVB on a PARPi resistant clone (MDA-MB-436-R), previously derived from the BRCA1-mutated breast 
cancer cell line MDA-MB-436 (47). These resistant cells restored HR by two mechanismnamely, stabilization and increased expression of a mutant BRCA1 protein and loss of 53BP1- resulting in increased RAD51 loading. While highly resistant to olaparib, the MDAMB-436-R cells were NVB sensitive, like the parental cells (Fig. S9A-B). In addition, we studied the PARPi-resistant clone UWB1.289-YSR12 (derived from the BRCA1-null human ovarian cancer cell line UWB1.289), which acquired resistance to PARPi via stabilization of replication forks (48). Although highly resistant to olaparib, these cells also remained sensitive to NVB, like the parental cells (Fig. S9C-D), once again suggesting that acquired resistance to PARPi does not determine cross-resistance to NVB.

Another known mechanism of PARPi resistance in $B R C A 1 / 2^{-/-}$tumors in the clinic is somatic reversion of the BRCA1/2 genes (18). We next determined whether NVB could overcome PARPi resistance caused by BRCA2 reversion. Unlike other PARPi resistant cells we analyzed, BRCA2-deficient tumor cells with near-perfect somatic reversion or CRISPRedited reversion of BRCA2 (PEO4 and CAPAN1-CR) $(49,50)$ were not only resistant to PARPi but also to NVB, when compared to their HR-deficient parental cell lines (PEO1 and CAPAN1) (Fig. S10A-D). These data suggest that NVB can overcome some but not all mechanisms of PARPi resistance.

\section{POL $\theta$ expression is a predictive biomarker for NVB sensitivity}

POL $\theta$ mRNA expression is increased in HR-deficient tumors and in several other cancers associated with poor prognosis $(17,21)$. We asked whether increased POL $\theta$ mRNA and protein expression may serve as a predictive biomarker for NVB sensitivity. As expected, CRISPR-mediated knockout of BRCA1 in RPE-1 cells resulted in increased POL $\theta$ expression as compare to the parental cells (Fig. 4E). Interestingly, all PARPi-resistant clones (R1-R4) retain high POL $\theta$ expression, revealing a direct correlation between POL $\theta$ 
expression and NVB sensitivity (Fig. 4E). This correlation was also present in the BRCA1deficient MDA-MB-436 cells. MDA-MB-436-R cells with acquired PARPi-resistance exhibited elevated POL $\theta$ mRNA expression and retained NVB sensitivity, while complementation of the parental cells with WT BRCA1 cDNA decreased POL $\theta$ expression and NVB sensitivity (Fig. 4F and S9A-B). More importantly, the direct correlation of POL $\theta$ expression and NVB sensitivity was observed in HR-deficient PDX models, where the elevated POL $\theta$ expression in DF83 and DF59 correlated with their high sensitivity to NVB in vivo (Fig. 3H and 3A-F).

Conversely, low POL $\theta$ expression was a predictive biomarker for NVB resistance. In the HR-proficient PDX cells (DF149) with wildtype BRCA1 and in the PARPi-resistant cell lines with BRCA2 reversion mutations (PEO4 and CAPAN1-CR), the cellular resistance to NVB was associated with low POLO expression levels (Fig. 3G and S10E). Also, the loss of POL $\theta$ in POLQ ${ }^{-/}$cells correlated with resistance to NVB (Fig. 2E). Taken together, in all cases examined, POL $\theta$ expression level correlated with cellular sensitivity to NVB, suggesting that POL $\theta$ expression in a tumor biopsy could provide a convenient predictive biomarker for patient enrollment in future clinical trials with NVB or other POL $\theta$ inhibitors.

\section{Toxic RAD51 foci is a mechanism of NVB cytotoxicity in PARPi resistant cells}

Depletion of POL $\theta$ in HR-deficient tumors resulted in RAD51 accumulation (17), which can be toxic if not regulated. Similarly, 53BP1 and POL $\theta$ are synthetic lethal, and the double knockout cells accumulate toxic RAD51 foci (46). We reasoned that accumulation of toxic RAD51 foci induced by NVB may be responsible for killing the PARPi resistant tumor cells. This is supported by the RAD51 pharmacodynamic results, where RAD51 foci were greatly increased in tumors after NVB treatment in vivo (Fig. 3G and S3D-F). We next determined RAD51 foci dynamics in our PARPi resistant cell line models. NVB induced 
accumulation RAD51 foci in PARPi-resistant RPE1-BRCA1/- clones (R1 and R4) more readily than in WT RPE1 cells after irradiation (Fig. 4G). Similarly, NVB treatment alone strongly induced RAD51 foci in the PARPi resistant MDA-MB-436-R cells (Fig. 4H). In addition, knockdown of RAD51 sensitized RPE1 WT cells to NVB but rescued BRCA1\% R1 clone from NVB cytotoxicity (Fig. 4I). Taken together, these results suggest toxic RAD51 foci, induced by NVB, is a mechanism for NVB cytotoxicity in HR-deficient cells with acquired PARPi resistance.

\section{DISCUSSION}

In summary, NVB is a specific and potent POL $\theta$ inhibitor which phenocopies POL $\theta$ depletion and is synthetic lethal in HR-deficient tumors. Many tumors types with PARPi resistance remain sensitive to NVB, depending on their mechanism of resistance. Tumors which acquire PARPi resistance by downregulating NHEJ and partially restoring HR repair remain sensitive to NVB. For these tumors, the persistent high level of POL $\theta$ expression is a predictive biomarker for their NVB responsiveness, and the NVB-induced RAD51 accumulation is both a pharmacodynamic biomarker and a mechanism of NVB cytotoxic activity.

Finally, our findings may translate to a potential new therapy for treating HR-deficient tumors in addition to PARP inhibition. Importantly, NVB, which is an oral and well-tolerated drug, has previously been investigated in a Phase 1 trial in which partial responses were documented, even though the enrolled patients were not preselected to have HR-deficient cancers (51). Results in this study provide a strong rationale for future clinical studies of NVB alone or in combination with PARPi to prevent or overcome PARPi resistance. 


\section{REFERENCES}

1. H. Farmer et al., Targeting the DNA repair defect in BRCA mutant cells as a therapeutic strategy. Nature 434, 917-921 (2005).

2. H. E. Bryant et al., Specific killing of BRCA2-deficient tumours with inhibitors of poly(ADP-ribose) polymerase. Nature 434, 913-917 (2005).

3. J. Ledermann et al., Olaparib maintenance therapy in platinum-sensitive relapsed ovarian cancer. $N$ Engl J Med 366, 1382-1392 (2012).

4. R. L. Coleman et al., Rucaparib maintenance treatment for recurrent ovarian carcinoma after response to platinum therapy (ARIEL3): a randomised, double-blind, placebo-controlled, phase 3 trial. Lancet 390, 1949-1961 (2017).

5. M. R. Mirza et al., Niraparib Maintenance Therapy in Platinum-Sensitive, Recurrent Ovarian Cancer. N Engl J Med 375, 2154-2164 (2016).

6. J. K. Litton et al., Talazoparib in Patients with Advanced Breast Cancer and a Germline BRCA Mutation. N Engl J Med 379, 753-763 (2018).

7. C. J. Lord, A. Ashworth, PARP inhibitors: Synthetic lethality in the clinic. Science 355, 1152-1158 (2017).

8. C. J. Lord, A. Ashworth, Mechanisms of resistance to therapies targeting BRCA-mutant cancers. Nat Med 19, 1381-1388 (2013).

9. W. Sakai et al., Secondary mutations as a mechanism of cisplatin resistance in BRCA2-mutated cancers. Nature 451, 1116-1120 (2008).

10. A. Ray Chaudhuri et al., Replication fork stability confers chemoresistance in BRCA-deficient cells. Nature 535, 382-387 (2016).

11. G. Xu et al., REV7 counteracts DNA double-strand break resection and affects PARP inhibition. Nature 521, 541-544 (2015).

12. S. M. Noordermeer et al., The shieldin complex mediates 53BP1-dependent DNA repair. Nature 560, 117-121 (2018).

13. H. Ghezraoui et al., 53BP1 cooperation with the REV7-shieldin complex underpins DNA structurespecific NHEJ. Nature 560, 122-127 (2018).

14. Y. J. He et al., DYNLL1 binds to MRE11 to limit DNA end resection in BRCA1-deficient cells. Nature 563, 522-526 (2018).

15. P. A. Mateos-Gomez et al., Mammalian polymerase theta promotes alternative NHEJ and suppresses recombination. Nature 518, 254-257 (2015).

16. G. S. Higgins, S. J. Boulton, Beyond PARP-POLtheta as an anticancer target. Science 359, 12171218 (2018).

17. R. Ceccaldi et al., Homologous-recombination-deficient tumours are dependent on Poltheta-mediated repair. Nature 518, 258-262 (2015).

18. P. A. Konstantinopoulos, R. Ceccaldi, G. I. Shapiro, A. D. D'Andrea, Homologous Recombination Deficiency: Exploiting the Fundamental Vulnerability of Ovarian Cancer. Cancer Discov 5, 1137-1154 (2015). 
19. D. M. Muzzini, P. Plevani, S. J. Boulton, G. Cassata, F. Marini, Caenorhabditis elegans POLQ-1 and HEL-308 function in two distinct DNA interstrand cross-link repair pathways. DNA Repair (Amst) 7, 941-950 (2008).

20. S. H. Chan, A. M. Yu, M. McVey, Dual roles for DNA polymerase theta in alternative end-joining repair of double-strand breaks in Drosophila. PLoS Genet 6, e1001005 (2010).

21. F. Lemee et al., DNA polymerase theta up-regulation is associated with poor survival in breast cancer, perturbs DNA replication, and promotes genetic instability. Proc Natl Acad Sci U S A 107, 1339013395 (2010).

22. G. S. Higgins et al., Overexpression of POLQ confers a poor prognosis in early breast cancer patients. Oncotarget 1, 175-184 (2010).

23. J. Zhou et al., Human CHD1 is required for early DNA-damage signaling and is uniquely regulated by its $\mathrm{N}$ terminus. Nucleic Acids Res 46, 3891-3905 (2018).

24. T. Yusufzai, J. T. Kadonaga, HARP is an ATP-driven annealing helicase. Science 322, 748-750 (2008).

25. M. Vedadi et al., Chemical screening methods to identify ligands that promote protein stability, protein crystallization, and structure determination. Proc Natl Acad Sci U S A 103, 15835-15840 (2006).

26. D. Martinez Molina et al., Monitoring drug target engagement in cells and tissues using the cellular thermal shift assay. Science 341, 84-87 (2013).

27. J. A. Newman, C. D. O. Cooper, H. Aitkenhead, O. Gileadi, Structure of the Helicase Domain of DNA Polymerase Theta Reveals a Possible Role in the Microhomology-Mediated End-Joining Pathway. Structure 23, 2319-2330 (2015).

28. K. Beagan et al., Drosophila DNA polymerase theta utilizes both helicase-like and polymerase domains during microhomology-mediated end joining and interstrand crosslink repair. PLoS Genet 13, e1006813 (2017).

29. M. J. Yousefzadeh, R. D. Wood, DNA polymerase POLQ and cellular defense against DNA damage. DNA Repair (Amst) 12, 1-9 (2013).

30. G. S. Higgins et al., A small interfering RNA screen of genes involved in DNA repair identifies tumorspecific radiosensitization by POLQ knockdown. Cancer Res 70, 2984-2993 (2010).

31. X. Liu et al., Somatic loss of BRCA1 and p53 in mice induces mammary tumors with features of human BRCA1-mutated basal-like breast cancer. Proceedings of the National Academy of Sciences 104, 12111-12116 (2007).

32. C. Pantelidou et al., PARP Inhibitor Efficacy Depends on CD8(+) T-cell Recruitment via Intratumoral STING Pathway Activation in BRCA-Deficient Models of Triple-Negative Breast Cancer. Cancer Discov 9, $722-737$ (2019).

33. T. Taniguchi et al., Disruption of the Fanconi anemia-BRCA pathway in cisplatin-sensitive ovarian tumors. Nat Med 9, 568-574 (2003).

34. K. S. Lim et al., USP1 Is Required for Replication Fork Protection in BRCA1-Deficient Tumors. Mol Cell 72, 925-941 e924 (2018).

35. A. Sugino, N. P. Higgins, P. O. Brown, C. L. Peebles, N. R. Cozzarelli, Energy coupling in DNA gyrase and the mechanism of action of novobiocin. Proc Natl Acad Sci U S A 75, 4838-4842 (1978). 
36. M. G. Marcu, A. Chadli, I. Bouhouche, M. Catelli, L. M. Neckers, The heat shock protein 90 antagonist novobiocin interacts with a previously unrecognized ATP-binding domain in the carboxyl terminus of the chaperone. J Biol Chem 275, 37181-37186 (2000).

37. T. Hsieh, D. Brutlag, ATP-dependent DNA topoisonmerase from D. melanogaster reversibly catenates duplex DNA rings. Cell 21, 115-125 (1980).

38. T. Goto, J. C. Wang, Yeast DNA topoisomerase II. An ATP-dependent type II topoisomerase that catalyzes the catenation, decatenation, unknotting, and relaxation of double-stranded DNA rings. $J$ Biol Chem 257, 5866-5872 (1982).

39. M. J. Pocklington, J. R. Jenkins, E. Orr, The effect of novobiocin on yeast topoisomerase type II. Mol Gen Genet 220, 256-260 (1990).

40. A. Drean, C. J. Lord, A. Ashworth, PARP inhibitor combination therapy. Crit Rev Oncol Hematol 108, 73-85 (2016).

41. T. Popova et al., Ploidy and large-scale genomic instability consistently identify basal-like breast carcinomas with BRCA1/2 inactivation. Cancer Res 72, 5454-5462 (2012).

42. M. R. Mirza, S. Pignata, J. A. Ledermann, Latest clinical evidence and further development of PARP inhibitors in ovarian cancer. Ann Oncol 29, 1366-1376 (2018).

43. L. B. Alexandrov et al., Signatures of mutational processes in human cancer. Nature 500, 415-421 (2013).

44. E. Pujade-Lauraine et al., Olaparib tablets as maintenance therapy in patients with platinum-sensitive, relapsed ovarian cancer and a BRCA1/2 mutation (SOLO2/ENGOT-Ov21): a double-blind, randomised, placebo-controlled, phase 3 trial. Lancet Oncol 18, 1274-1284 (2017).

45. K. Parmar et al., The CHK1 Inhibitor Prexasertib Exhibits Monotherapy Activity in High-Grade Serous Ovarian Cancer Models and Sensitizes to PARP Inhibition. Clin Cancer Res, (2019).

46. W. Feng et al., Genetic determinants of cellular addiction to DNA polymerase theta. Nat Commun 10, 4286 (2019).

47. N. Johnson et al., Stabilization of mutant BRCA1 protein confers PARP inhibitor and platinum resistance. Proc Natl Acad Sci U S A 110, 17041-17046 (2013).

48. S. A. Yazinski et al., ATR inhibition disrupts rewired homologous recombination and fork protection pathways in PARP inhibitor-resistant BRCA-deficient cancer cells. Genes Dev 31, 318-332 (2017).

49. L. J. Barber et al., Comprehensive genomic analysis of a BRCA2 deficient human pancreatic cancer. PLoS One 6, e21639 (2011).

50. W. Sakai et al., Secondary mutations as a mechanism of cisplatin resistance in BRCA2-mutated cancers. Nature 451, 1116-1120 (2008).

51. J. P. Eder, C. A. Wheeler, B. A. Teicher, L. E. Schnipper, A phase I clinical trial of novobiocin, a modulator of alkylating agent cytotoxicity. Cancer Res 51, 510-513 (1991). 


\section{ACKNOWLEDGEMENTS}

We thank the ICCB-Longwood Screening Facility at Harvard Medical School, for their help in small-molecule screening. We thank Prafulla Gokhale and Qing Zeng at Belfer Center for

Applied Cancer Science for their help in PDX studies. We thank Connor Clairmont for providing RPE BRCA1\% cells and Lisa Moreau for her help in chromosome aberration assays. We thank Drs. Huy Nguyen and Sandor Spisak for genomic analysis of the POL $\theta$ Knockout clones. We thank Dr. Jeremy Stark at Beckman Research Institute of the City of Hope for providing DR-GFP and EJ-2 repair substrates.

\section{FUNDING}

This research was supported by a Stand Up To Cancer (SU2C)-Ovarian Cancer Research Fund Alliance-National Ovarian Cancer Coalition Dream Team Translational Research Grant (SU2C-AACR-DT16-15) to A.D.D. SU2C is a program of the Entertainment Industry Foundation. Research grants are administered by the American Association for Cancer Research, the scientific partner of SU2C. This work was also supported by the NIH (grants R37HL052725, P01HL048546, P50CA168504), the US Department of Defense (grants BM110181 and BC151331P1), as well as grants from the Breast Cancer Research Foundation and the Fanconi Anemia Research Fund to A.D.D., as well as the ERC starting grant ( $\left.N^{\circ} 714162\right)$ and the Ville de Paris Emergences Program grant ( $N^{\circ}$ DAE 137) to R.C. This work was also supported by Ann Schreiber Mentored Investigator Award from Ovarian Cancer Research Fund Alliance (457527) and Joint Center for Radiation Therapy Award from Harvard Medical School to J.Z. J.A.T. and A.S. are supported by NIH grants P01 CA092548, R35 CA220430, and by the Cancer Prevention and Research Institute of Texas and the Robert A. Welch Chemistry Chair. 


\section{COMPETING INTERESTS}

A.D. D'Andrea reports receiving commercial research grants from Eli Lilly \& Company, Sierra Oncology, and EMD Serono and is a consultant/advisory board member for Eli Lilly

\& Company, Sierra Oncology, and EMD Serono. G.I. Shapiro has received research funding from Eli Lilly, Merck KGaA/EMD-Serono, Merck, and Sierra Oncology, and he has served on advisory boards for Pfizer, Eli Lilly, G1 Therapeutics, Roche, Merck KGaA/EMD-Serono, Sierra Oncology, Bicycle Therapeutics, Artios, Fusion Pharmaceuticals, Cybrexa Therapeutics, Astex, Almac, Ipsen, Bayer, Angiex, Daiichi Sankyo, Boehringer Ingelheim, ImmunoMet and Asana.

\section{DATA AND MATERIALS AVAILABILITY}

All related data have been presented in the main manuscript or in supplementary data.

\section{SUPPLEMENTARY MATERIALS}

Figs. S1 to $\mathrm{S} 10$

Legends for Figs. S1 to S10

Materials and Methods

Supplementary References 


\section{FIGURE LEGENDS}

Figure 1. A small-molecule screen identifies Novobiocin (NVB) as a specific POL $\theta$ ATPase inhibitor. A, The domain structures of full length (FL) and ATPase domain (or $\triangle$ Pol, a.a. 1-987) of POL $\theta$, a Coomassie-stained gel of the purified POL $\theta$ ATPase domain, and a schematic of the small molecule screen for POL $\theta$ inhibitors. B, Results of the small-molecule screen. Shown is quantification of POLO ATPase activity measured by the ADP-Glo assay in the presence of small-molecule libraries. We screened a total of 23,883 small-molecules in duplicate across multiple libraries enriched with known bioactive compounds, and we identified 72 compounds $(0.3 \%$ of total) that significantly reduced POL $\theta$ ATPase activity ( $z$ score < -4). Four top hits verified in the secondary screen (Fig. S1B-E) were labeled; Mitoxantrone (MTX), Suramin (SUR), Novobiocin (NVB) and Aurintricarboxylic acid (AUR). Data shown are mean \pm s.e.m, $n=2$. C, Quantification of POL $\theta$ and SMARCAL1 ATPase activity in the presence of indicated small-molecules. Mean \pm s.d., $n=3$. D, Pulldown experiments in vitro with NVB-conjugated beads and purified POL $\theta$ and SMARCAL1 ATPase domains (Flag-tag purification from SF9 cells). Input and pulldown proteins were detected by Coomassie staining on SDS gel. E, Thermal shift assay with NVB at indicated temperatures. Full length GFP-POL $\theta$ was detected by Western blot using the GFP antibody and PARP1 by a PARP1 antibody. Data in $\mathbf{D}$ and $\mathbf{E}$ are representative images of three independent experiments. F, The NVB binding tunnel in POLQ ATPase domain (shown in surface - PDB 5AGA) was predicted by extra precision glide docking and lowest binding free energy from prime MM-GBSA calculations to have multiple hydrogen bonds and close hydrophobic packing. Top and side views showing NVB docking into the tunnel. Binding modes of NVB (green sticks) and AMP-PNP (magenta sticks) in POLQ helicase domain (PDB 5AGA) with green sphere showing active site $\mathrm{Mg}^{2+}$ ion. G, Representative images and quantification of POL $\theta$ accumulation at sites of laser micro-irradiated DNA damage in cells overexpressing GFP-tagged, full-length POL $\theta$ in the presence of indicated inhibitors. Mean \pm s.e.m, $n=3$. H, MMEJ repair reporter assay in U2OS cells treated with indicated inhibitors. Mean \pm s.d., $n=3$. Statistics analysis between groups was performed using $t$-test with Welch's correction. *,$p<0.05 ;{ }^{* *}, p<0.01 ;{ }^{* * *}, p<0.001$; ns, not significant. 
Figure 2. NVB kills HR-deficient tumors in vivo and in vitro and synergizes with PARPi. A, Tumor growth of the GEMM model (Brca1\%- TNBC) after treatment with vehicle (PBS) or NVB. Tumor chunks from GEMM mice were implanted in syngeneic FVB/129P2 mice, which were treated with PBS or $100 \mathrm{mg} / \mathrm{kg}$ NVB via IP injection twice a day for 5 weeks. $n=6$ for PBS group and $n=7$ for NVB group. Mean \pm s.e.m. is shown. Statistical analyses were $t$-test. B, Survival plot of the experiment shown in $(\mathbf{A})$. Median survival and $p$ value are shown. Statistics analyses were $t$-test. $\mathbf{C}$, Tumor growth of the xenograft model with TOV21G (FANCF deficient ovarian cancer) or FANCF-complemented TOV21G cells. The tumor bearing mice were treated with vehicle or $100 \mathrm{mg} / \mathrm{kg}$ NVB via IP injection twice a day for 4 weeks. Fold change of tumor was calculated using the formula Fold = T/T0, where $\mathrm{T}$ is the tumor size at given time and T0 is the initial tumor size. Data are Mean \pm s.e.m., $\mathrm{n}=10$ in each group. Dunn's multiple comparisons test. Representative images are TOV21G xenograft tumors at the end of NVB treatment. D, Clonogenic survival of $B R C A 1^{-}$ /- and WT RPE1 cells under increasing concentrations of the POL $\theta$ inhibitor NVB. Survival is normalized to the untreated (Unt) sample. Data shown are mean \pm s.e.m., $n=4$. E, Representative images and quantification of clonogenic survival of $P O L Q^{-/-}$and WT U2OS cells under increasing concentrations of NVB. Survival is shown as relative to the untreated (Unt) sample. Data shown are mean $\pm \mathrm{SD}, \mathrm{n}=5$. F, Clonogenic survival of $B R C A 1^{-/}$and WT RPE1 cells under increasing concentrations of the PARPi Rucaparib alone or in combination with NVB. Quantifications, representative images and calculated IC50 values are shown. Data shown are mean \pm s.e.m., $n=3$. 
Figure 3. NVB overrides PARPi resistance in vivo in PDX models and RAD51 is a dynamic pharmacodynamic biomarker. A-B, Efficacy of NVB in PDX model DF83 (RAD51C deficient). NSG mice ( $n=7$ mice/group) bearing luciferized DF83 cells derived from an ovarian cancer patient were treated with $50 \mathrm{mg} / \mathrm{kg}$ of olaparib (daily, orally), 75 $\mathrm{mg} / \mathrm{kg}$ NVB (via IP, b.i.d.), or both for 4 weeks. Tumor growth was monitored weekly by bioluminescence imaging. Representative images of tumor burden on day 0 and day 28 are shown, and quantifications shown are mean \pm s.e.m. $\log _{2}$ (fold change in tumor size) was calculated using the formula $\log _{2}(\mathrm{~T} / \mathrm{T} 0)$, where $\mathrm{T}$ is the tumor volume at given time and T0 is the initial tumor volume. C-D, Efficacy of NVB in PARPi resistant PDX model DF59 (BRCA1-deficient, 53BP1 loss), $\mathrm{n}=6 \mathrm{mice/group.} \mathrm{Same} \mathrm{conditions} \mathrm{and} \mathrm{analysis} \mathrm{were}$ applied as in A-B. E-F, Efficacy of NVB in PARPi resistant PDX model DF149 (BRCA1-WT, HR proficient), $n=7$ mice/group. Same conditions and analysis were applied as in $\mathbf{A}-\mathbf{B}$. G, Representative IHC images and quantifications of RAD51 foci in DF-59 and DF-83 PDX models. Tumor bearing mice were dosed with indicated drugs and as in efficacy study in Fig 3 , and tumor cells were collected from ascites 52 hours after first dose. FFPE sections of the isolated tumor cells were stained with a RAD51 antibody. The percentage of RAD51foci positive cells (> 4 foci/cell) in 3 random 40X fields was estimated in DF-59. No RAD51 focus was observed in the DF-83 model. Statistical analysis method was one-way ANOVA Bonferroni's multiple comparisons test. ${ }^{*}, p<0.05$; ${ }^{* *}, p<0.01$; ${ }^{* \star *}, p<0.0001$. H. POLQ expression at mRNA level in PDX models DF53, DF83 and DF149. 
Figure 4. POLQ expression level is a predictive biomarker for NVB sensitivity. A. A schematic shows how the PARPi resistant clones R1-R4 were generated. TP53 was knocked out in order to generate BRCA1 knockout (BRCA1/-) RPE1 cells, which were then used for selection of PARPi resistant clones. B. A Western blot of WT and BRCA1/- RPE1 cells and the PARPi resistant clones, using an anti-BRCA1 antibody (Millipore \#OP92). No BRCA1 reversion was observed in the clones. C-D. NVB sensitivity of WT RPE1, BRCA1\% RPE1, and PARPi-resistant $B R C A 1^{-/}$clones (R1-R4). R1 was selected by olaparib and R2R4 were selected by Niraparib. R1 and R2 were tested in clonogenic survival assay (C). R3 and R4 were tested in CellTiter-Glo cell viability assay (D). Data are mean \pm s.e.m., $n=3$. E. POLQ expression at mRNA level and protein level in WT RPE1, BRCA1/- RPE1, and PARPi-resistant $B R C A 1^{-}$clones (R1-R4). POLQ mRNA was measured by qRT-PCR and normalized to beta-Actin (ACTB). F. POLQ expression at mRNA level in parental (P), PARPi resistant $(\mathrm{R})$, and empty vector (+EV) or BRCA1-cDNA (+BR1) complemented MDA-MB436 cells. G, RAD51 foci assay with DMSO or NVB in RPE1-BRCA1 WT RPE1, BRCA1\%RPE1, and PARPi-resistant BRCA1\%-clones (R1-R4) 4 hours after 5 Gy of IR. Cells with more than 10 foci were counted as positive. Data were Mean \pm SEM from two independent experiments. H, RAD51 foci assay with DMSO or NVB in MDA-MB-436 or the PARPiresistant MDA-MB-436-R. Cells with more than 5 foci were counted as positive. Data were Mean \pm SEM from two independent experiments. I. Novobiocin sensitivity of RPE1-WT and the R1 PARPi-resistant clone after being treated with Control or RAD51 siRNA. A Western blot shows the efficiency of RAD51 knockdown in RPE1-WT and the R1 PARPi-resistant clone. 
Fig 1

A

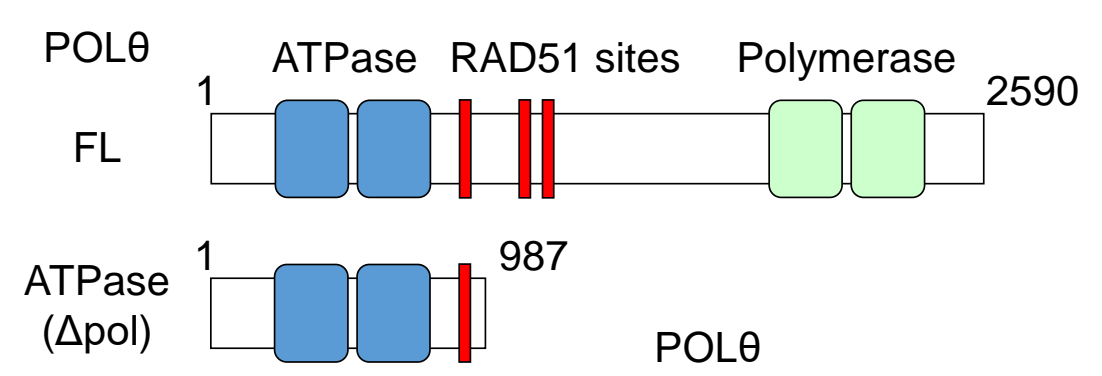

B

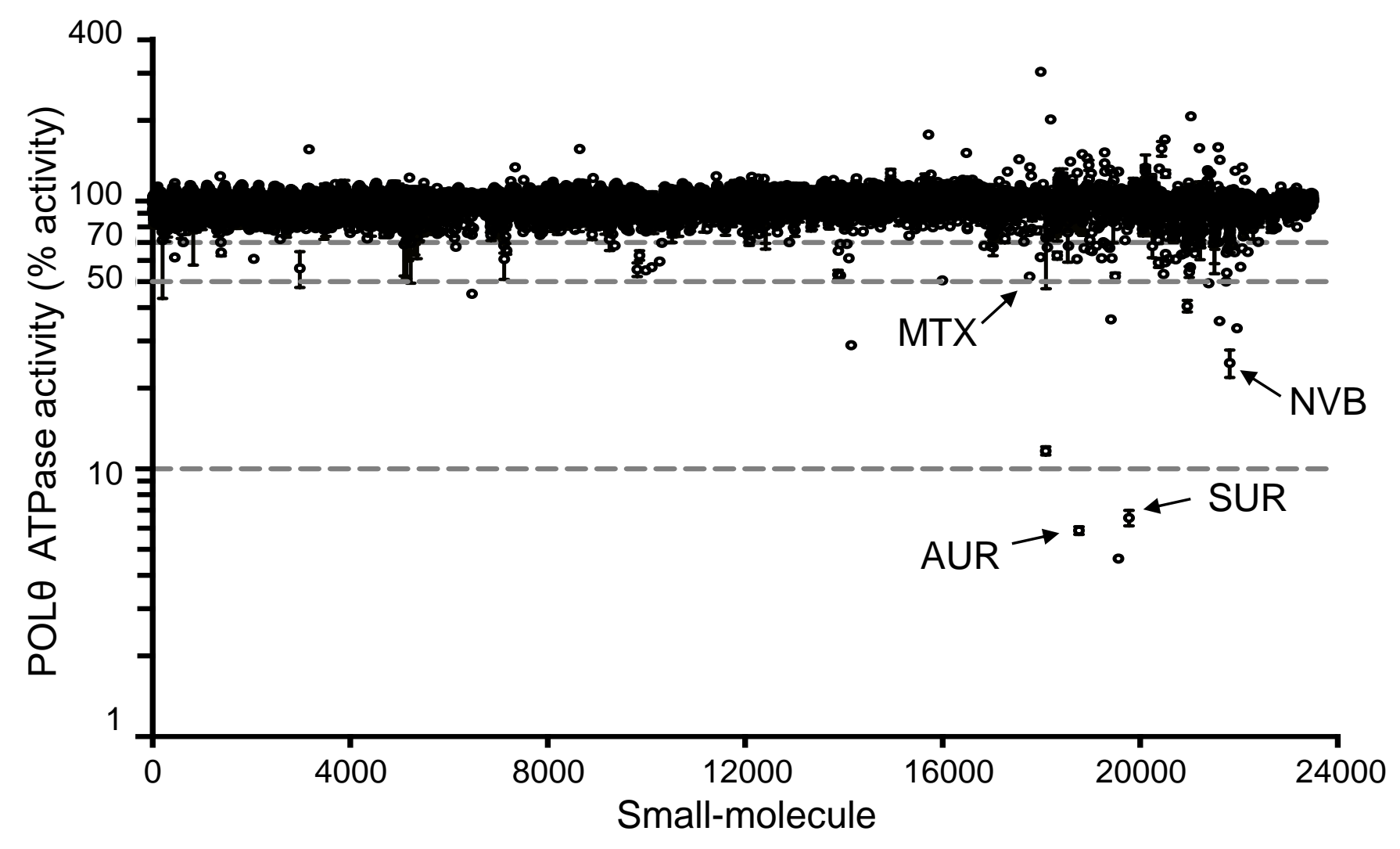

C

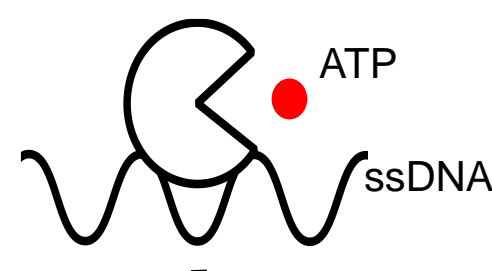

Small-molecule POL $\theta$
$1-987$ ADP-Glo librairies

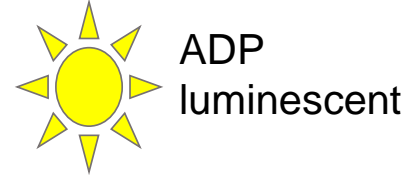

E

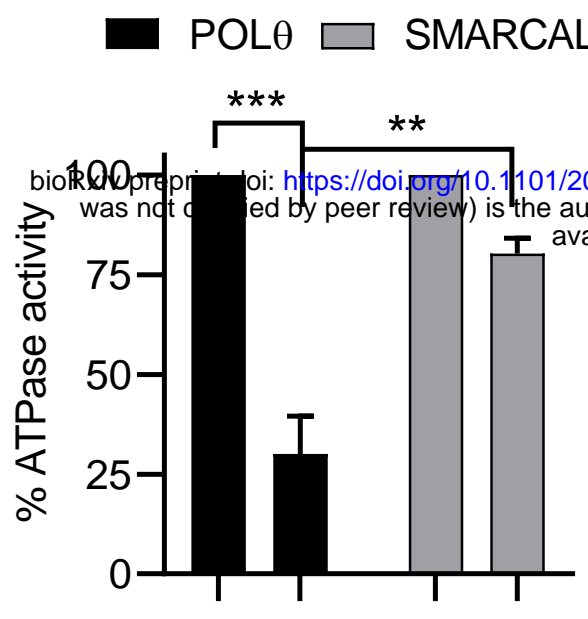

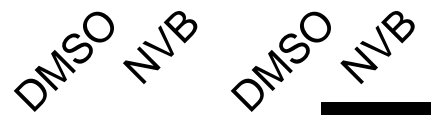

Purified anted bio Bxiv a license to display the preprint in perpetuity It is made

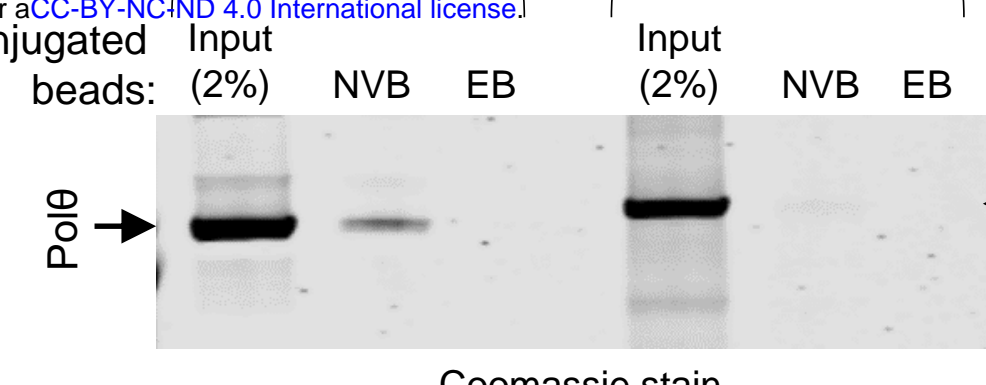

Coomassie stain
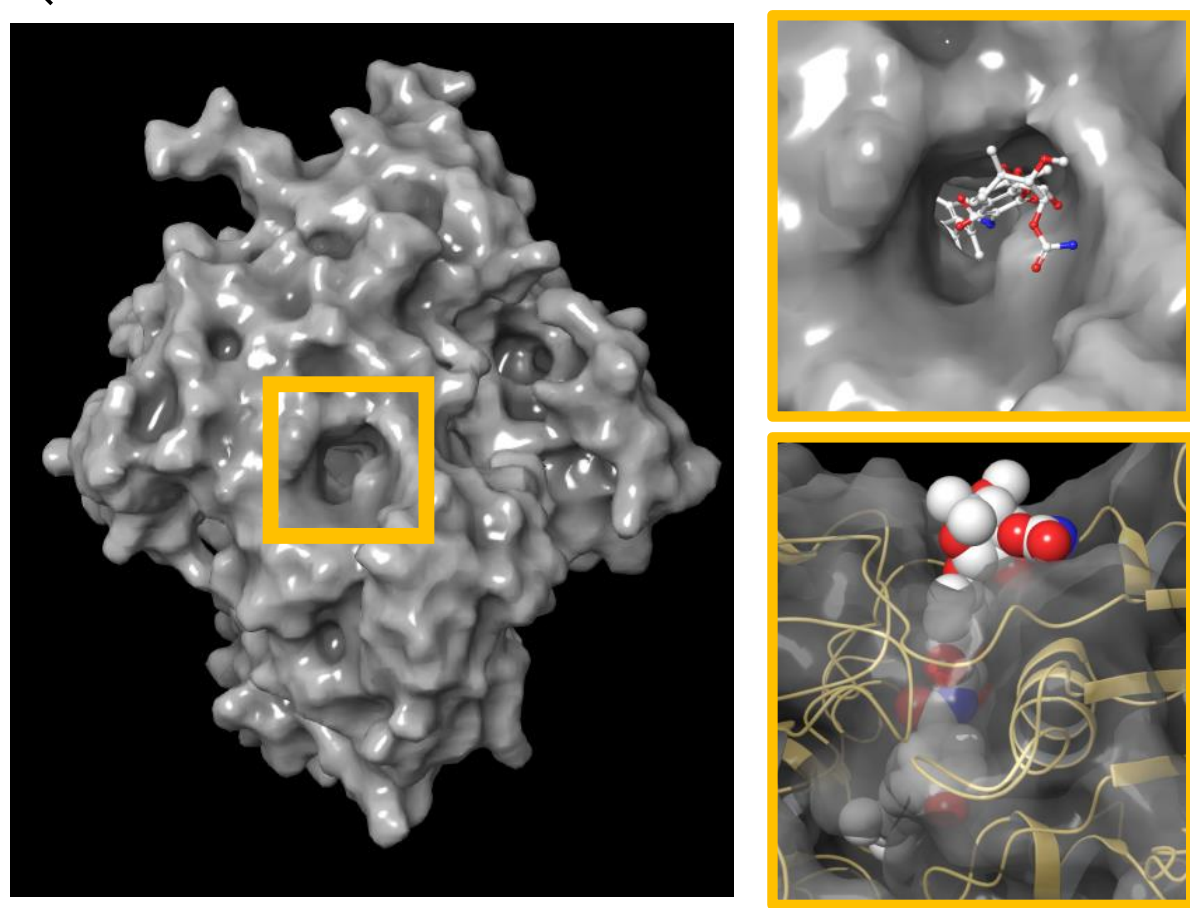

G

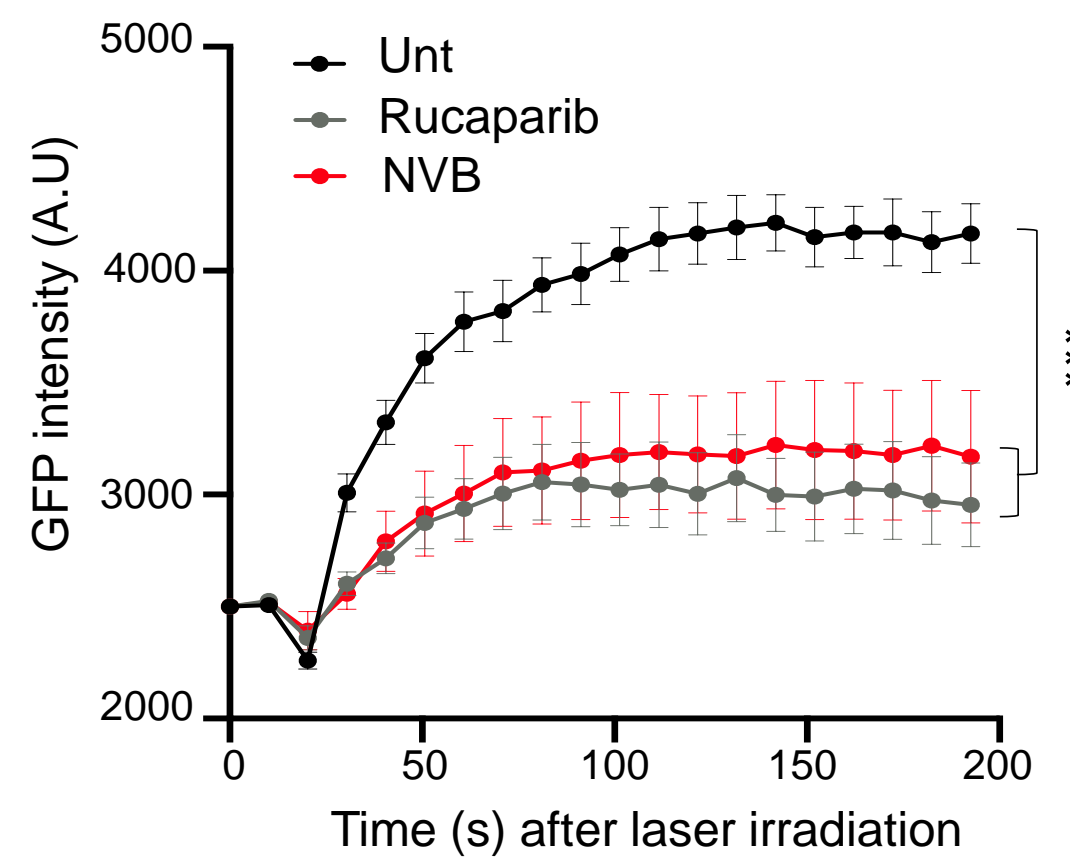

Laser irradiation

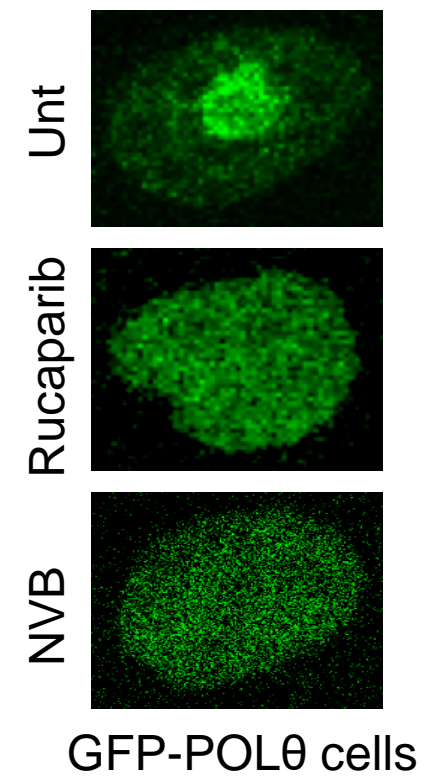

Temp $\left({ }^{\circ} \mathrm{C}\right)$ :

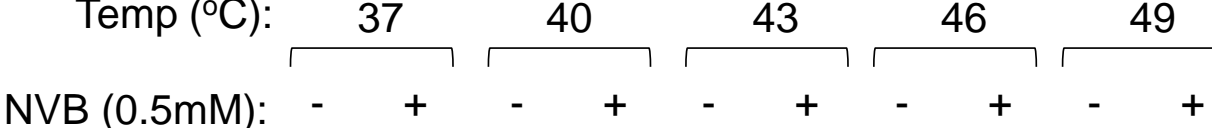
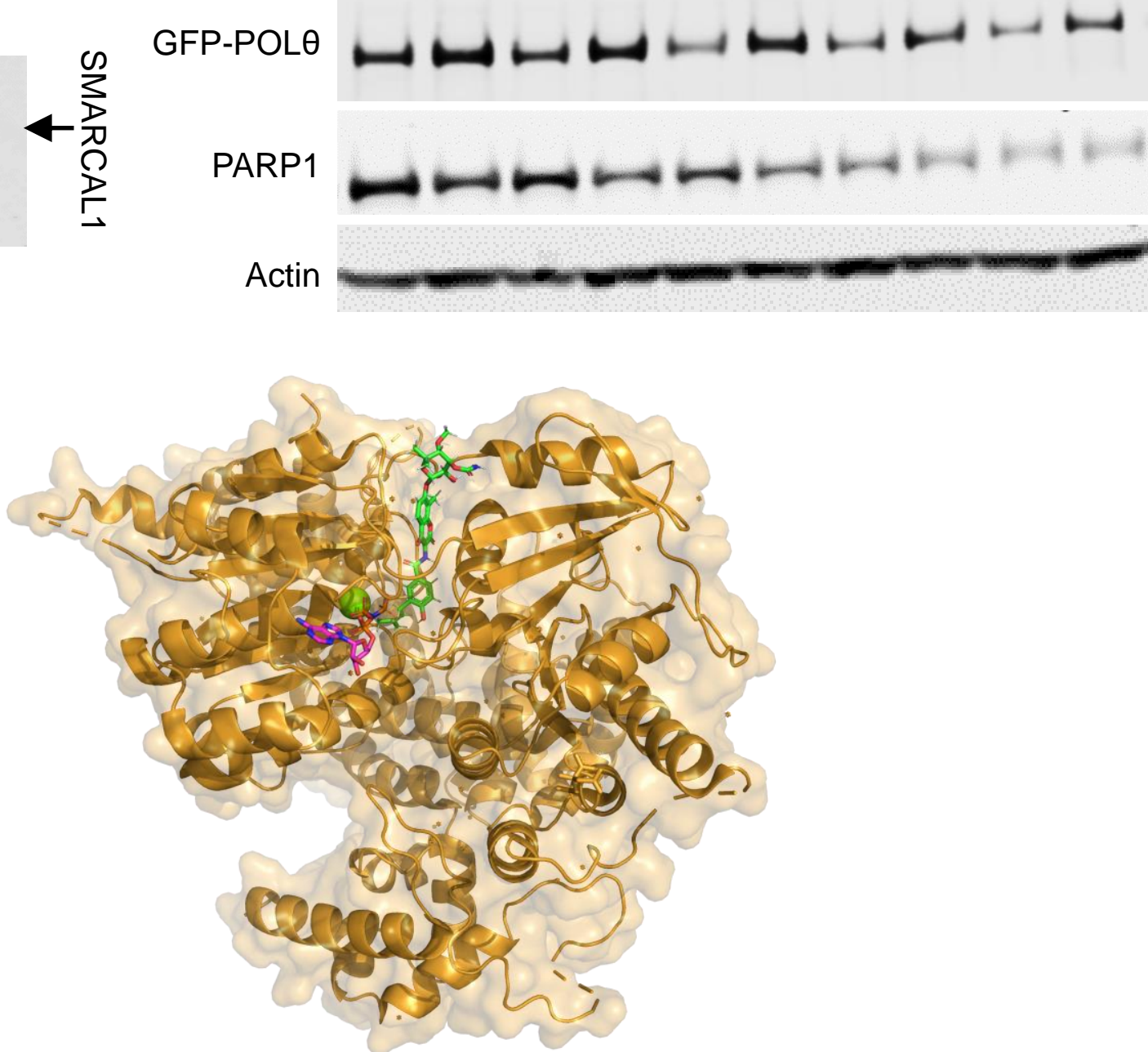

H

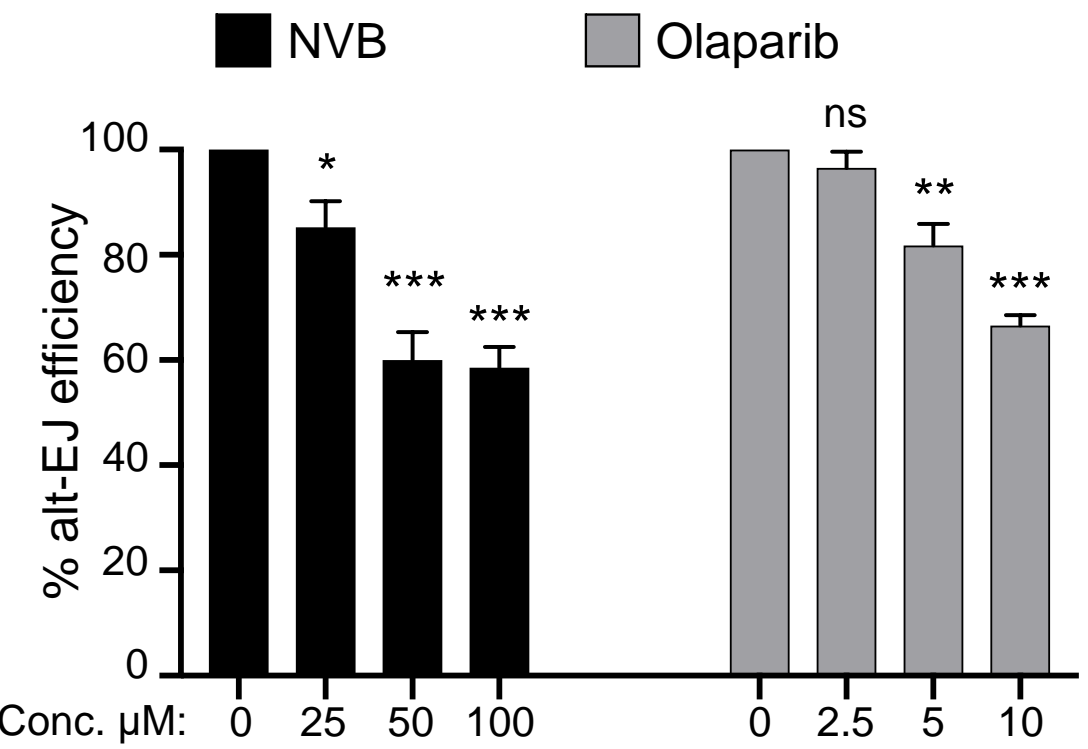


Fig 2

A

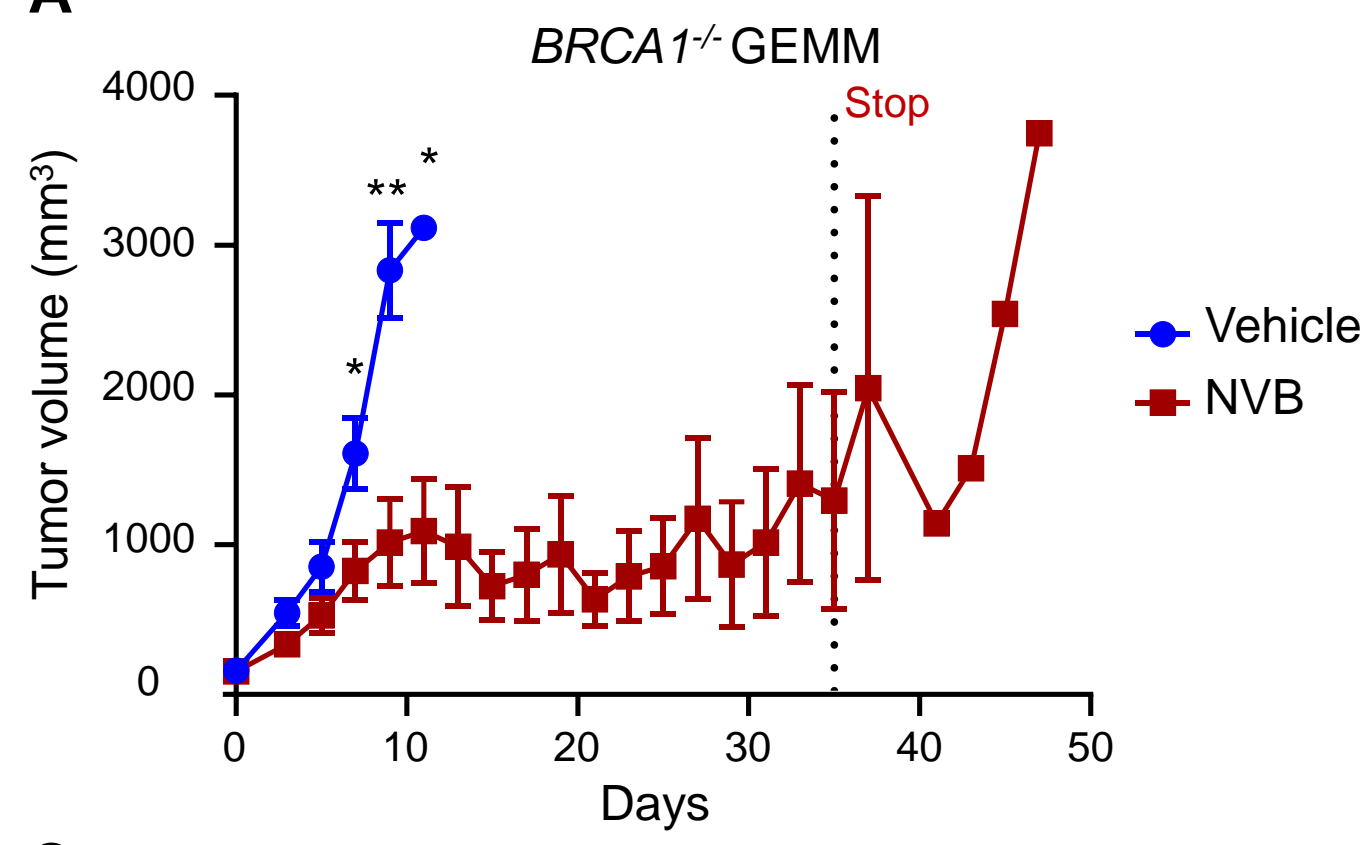

c

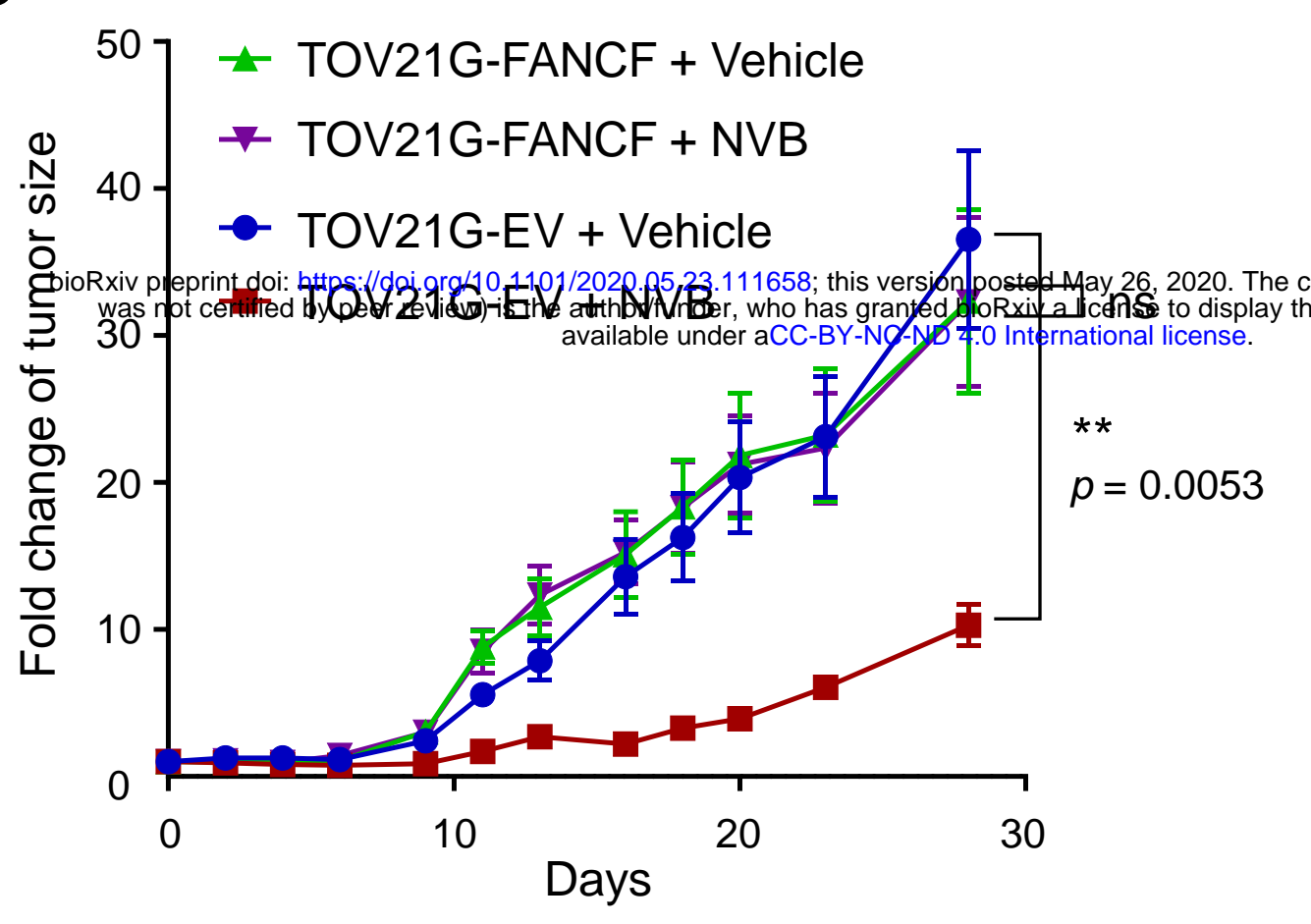

B

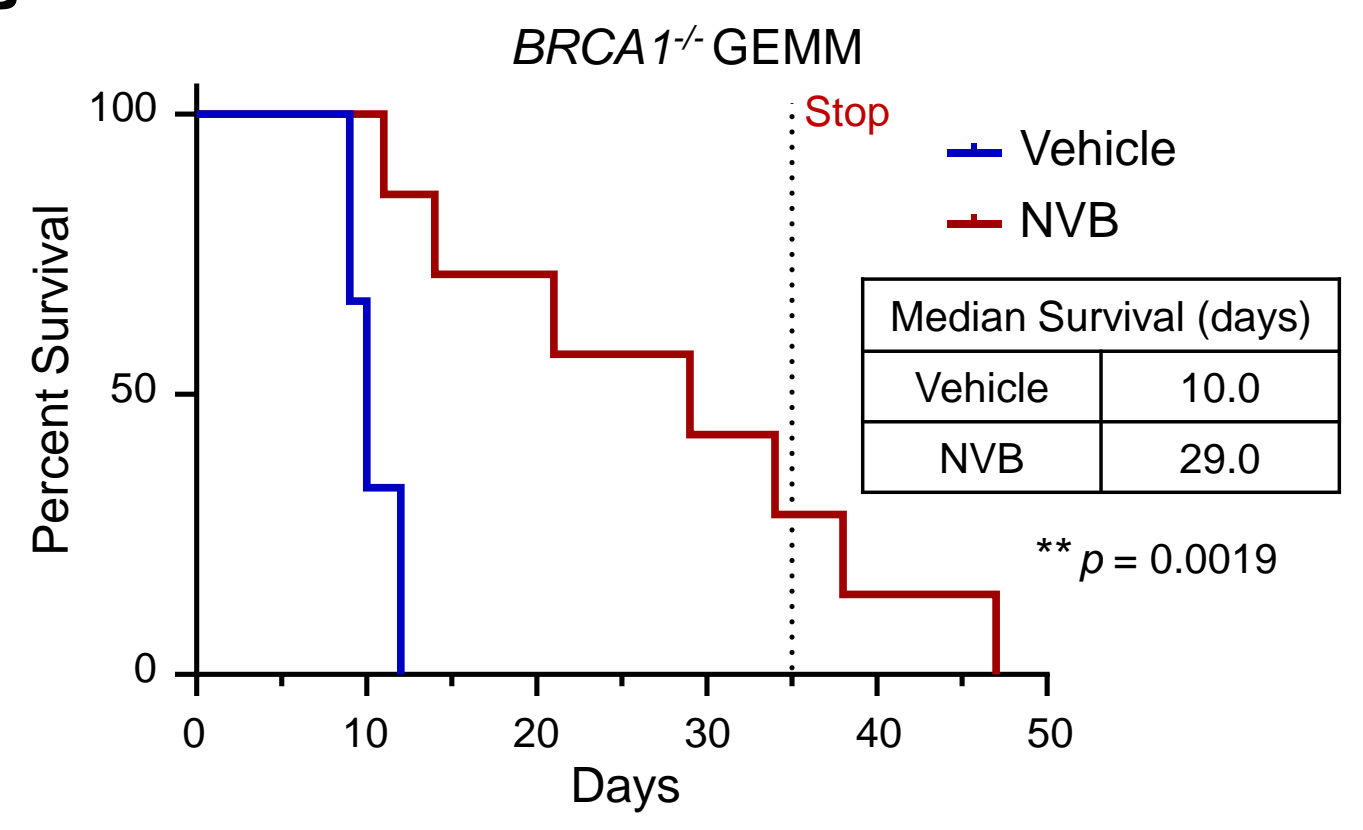

Treatment:

Vehicle

NVB

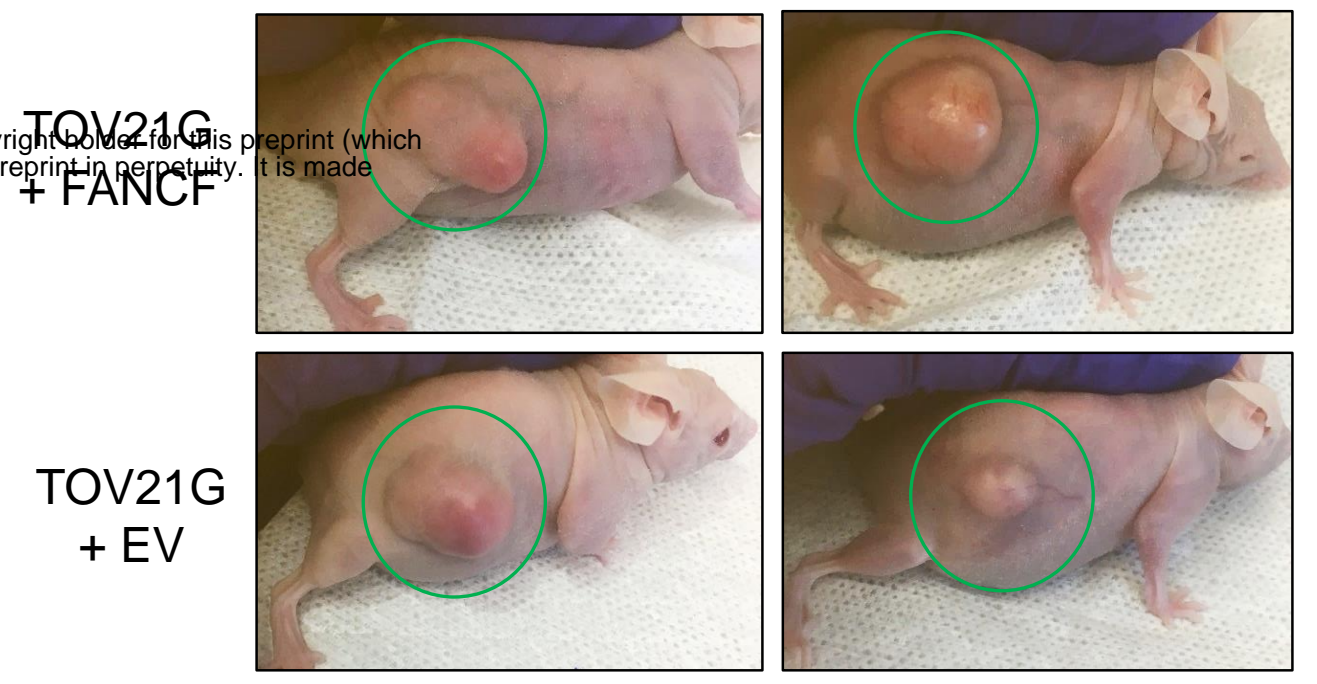

D

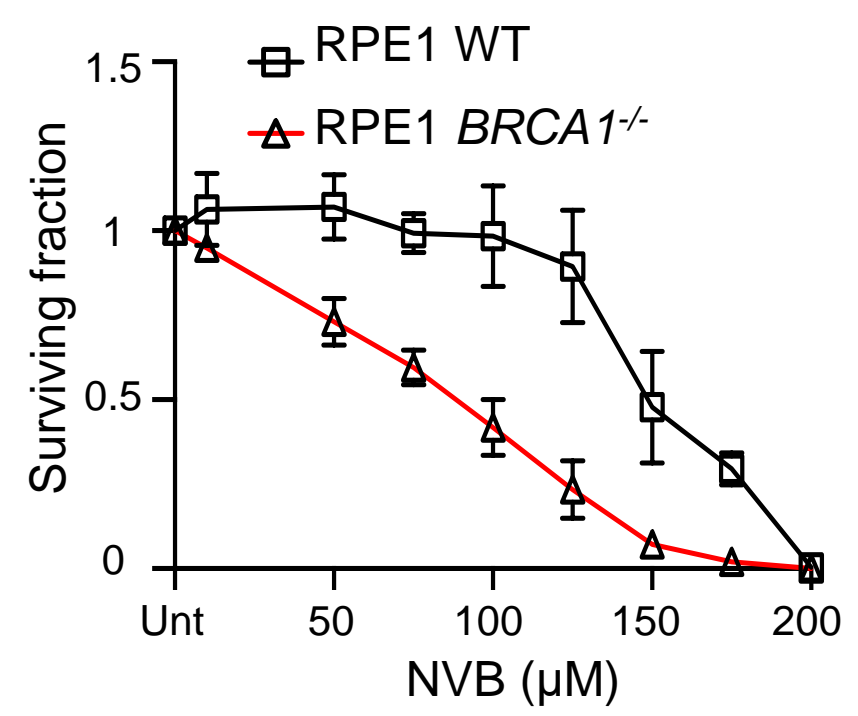

E

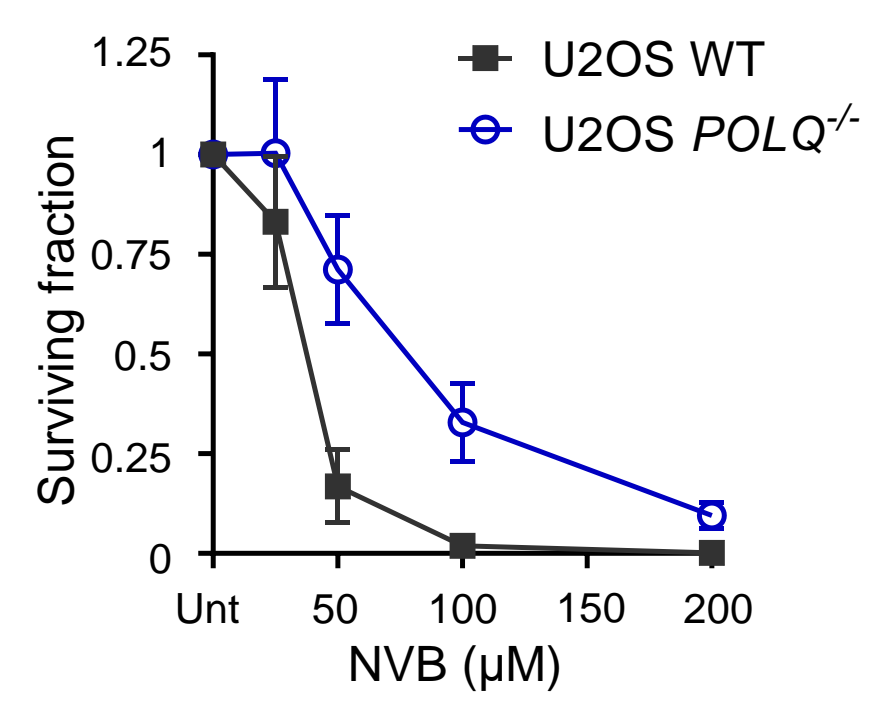

NVB $(\mu \mathrm{M})$

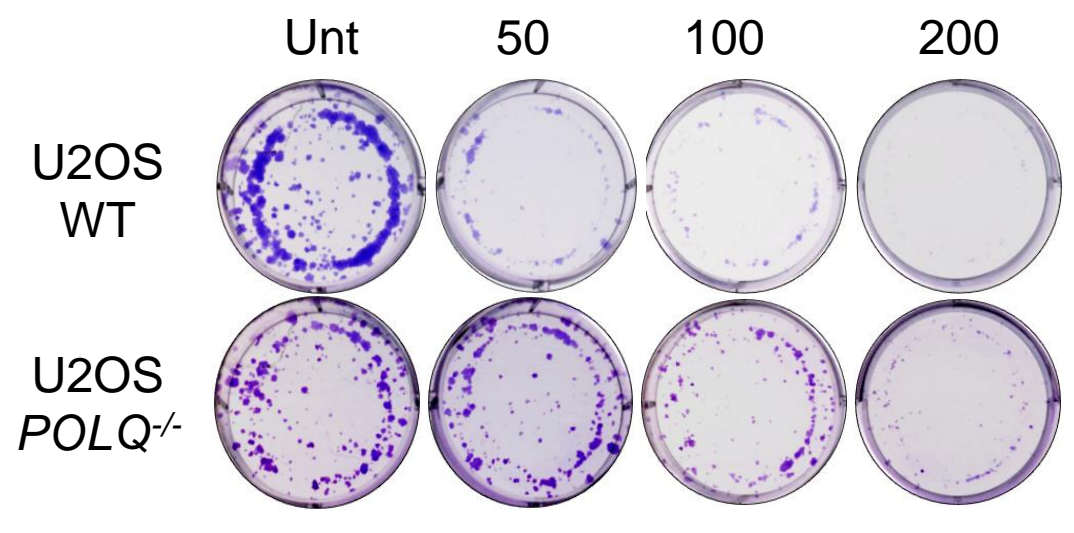

F
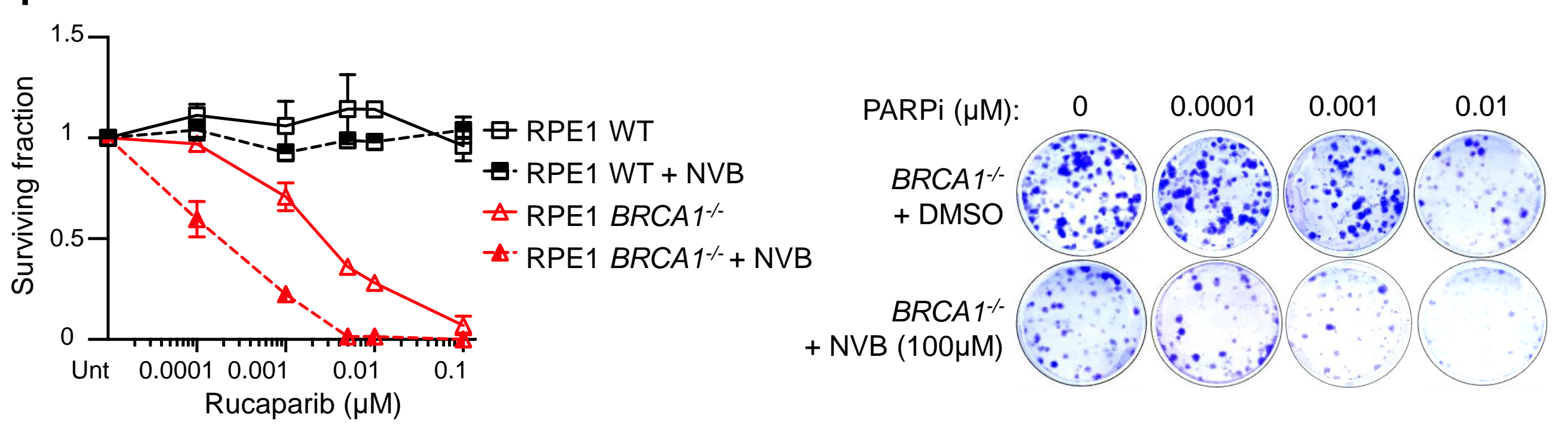

RPE1 BRCA1\%

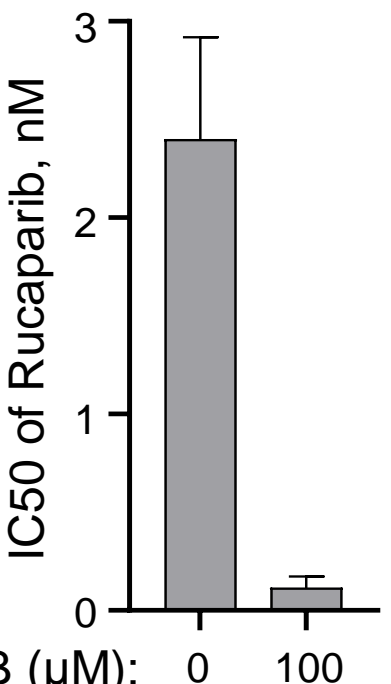


Fig 3

A

PDX DF83 ( $\triangle$ RAD51C)

Day 0

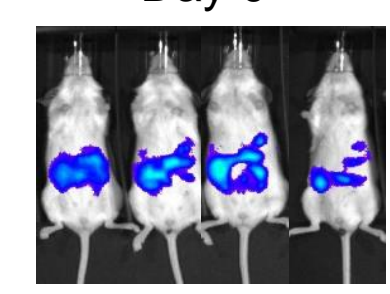

Day 28

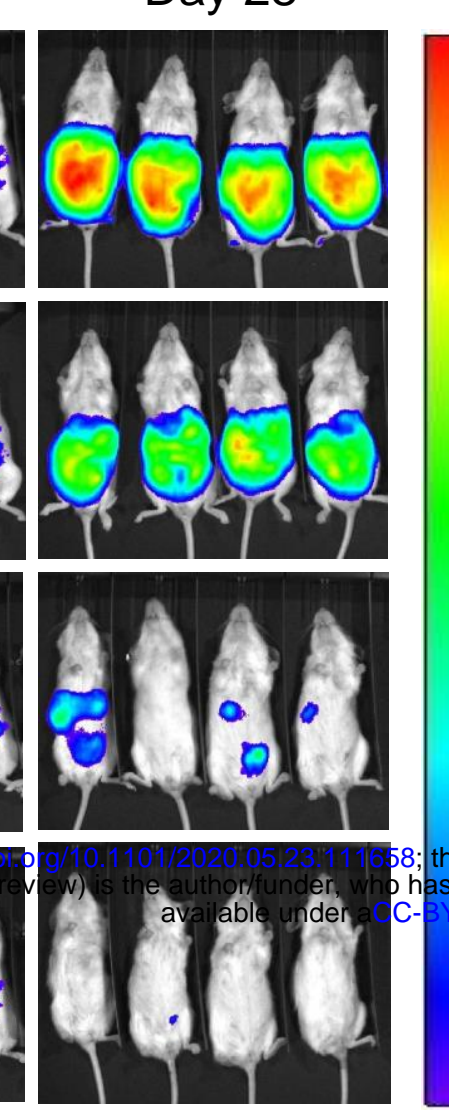

Radiance

(p/sec/cm²/sr)
B

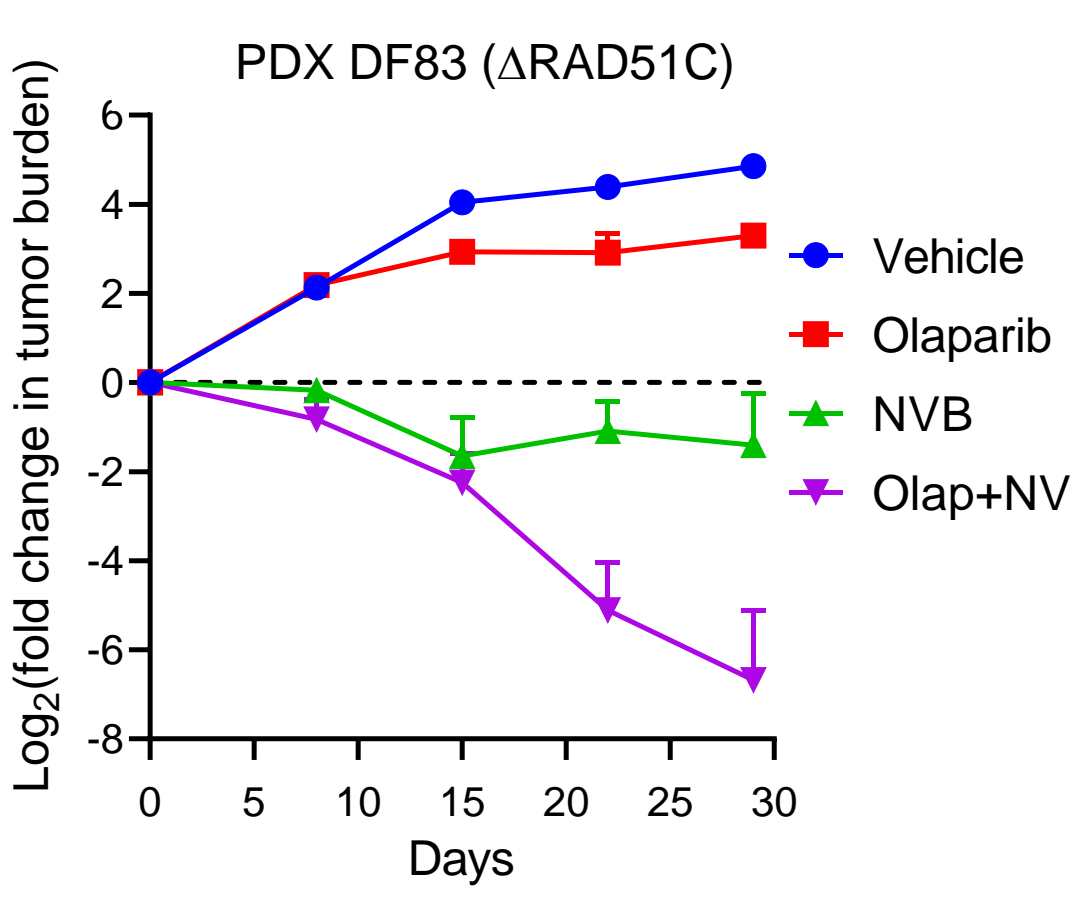

G

DF-59
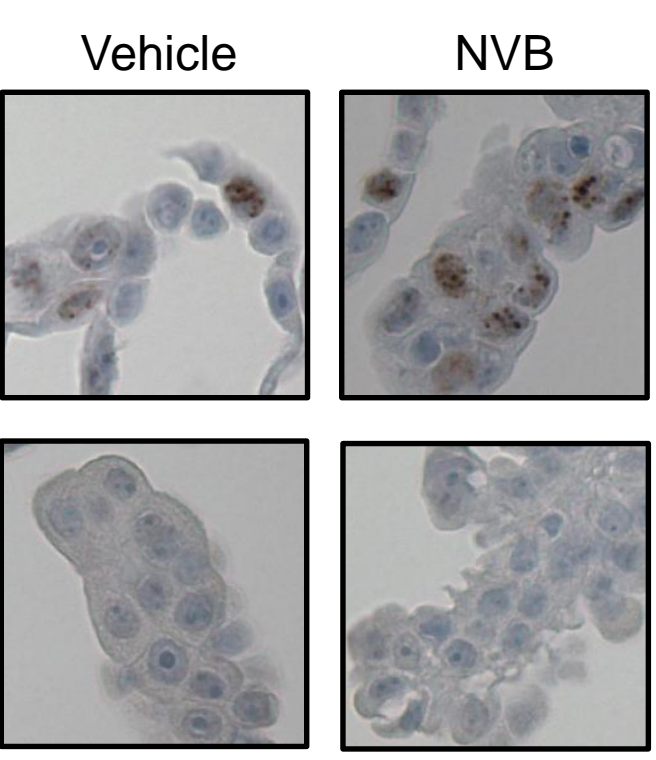

C

PDX DF59 (ABRCA1; $\triangle 53 \mathrm{BP} 1)$

Day 0

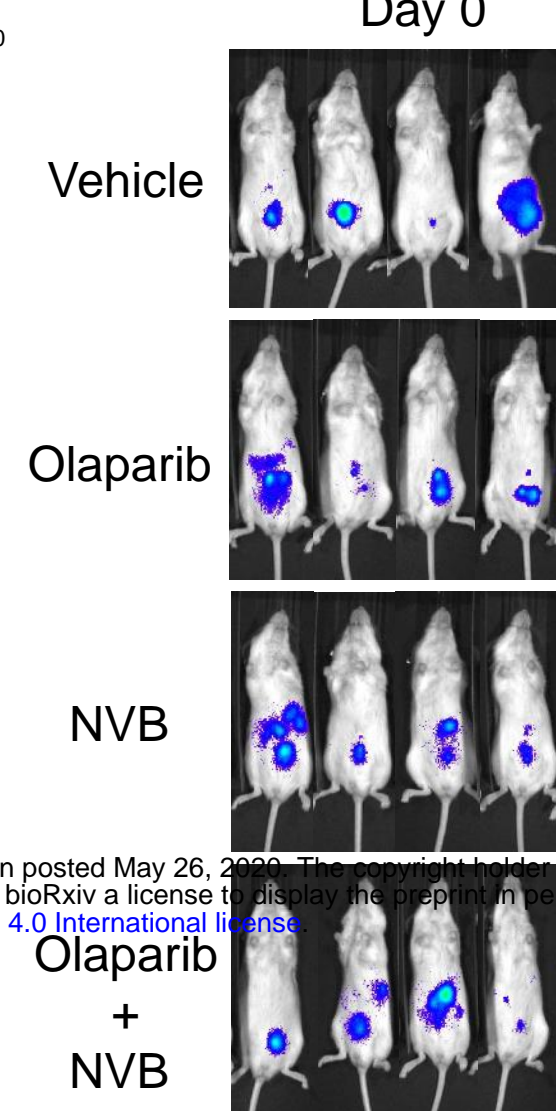

D

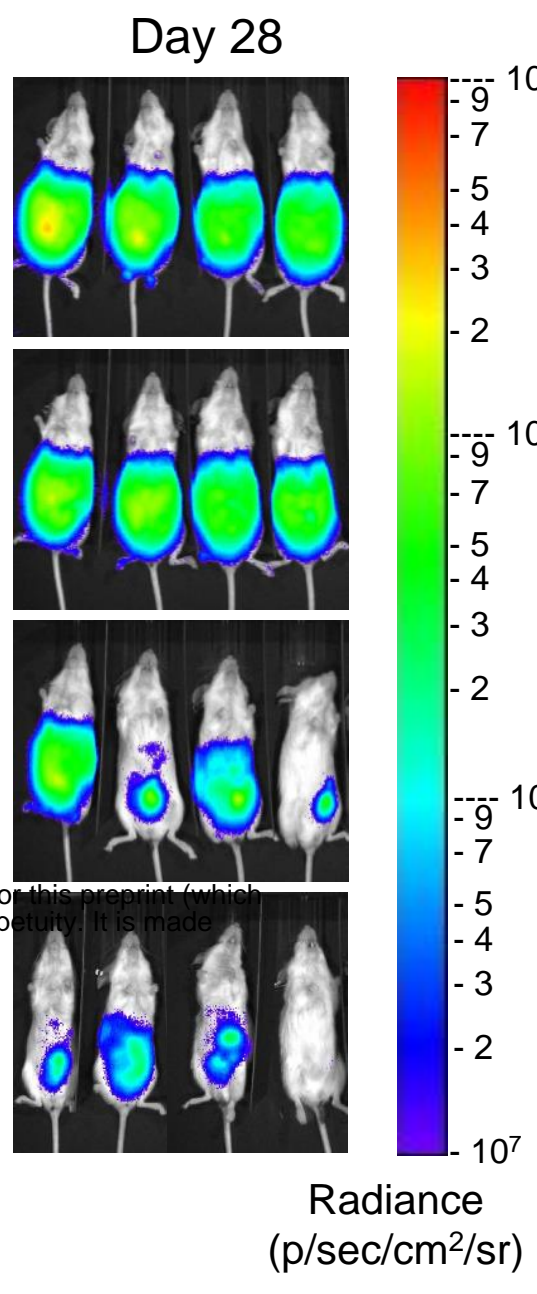

E

PDX DF149 (HR proficient)
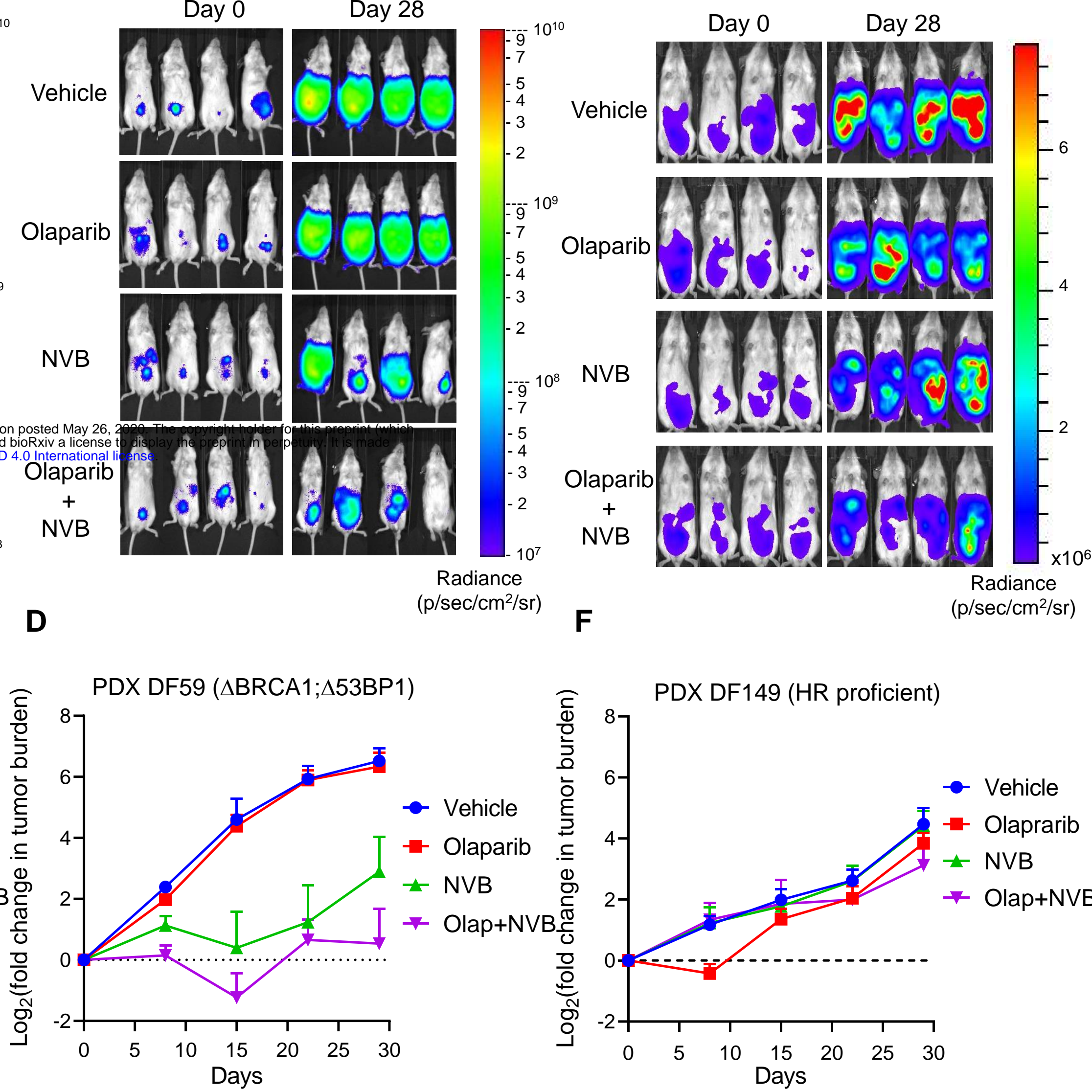

H

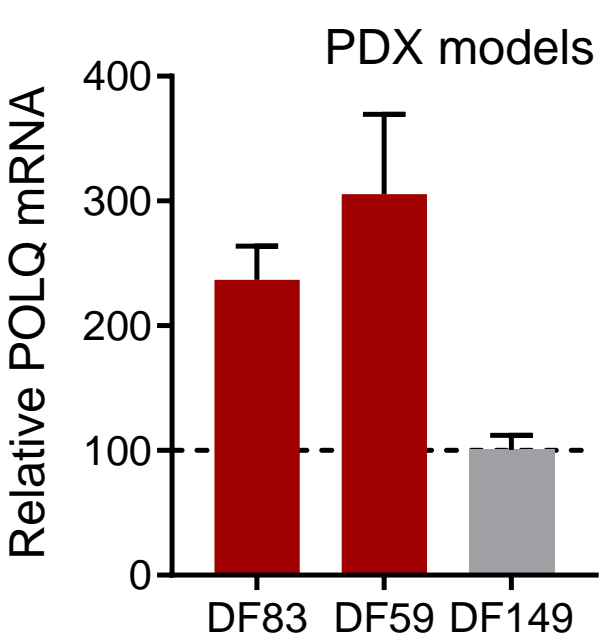

NVB sensitivity: $S \quad S \quad R$ PARPi sensitivity: $S \quad R \quad R$ 
Fig 4

A

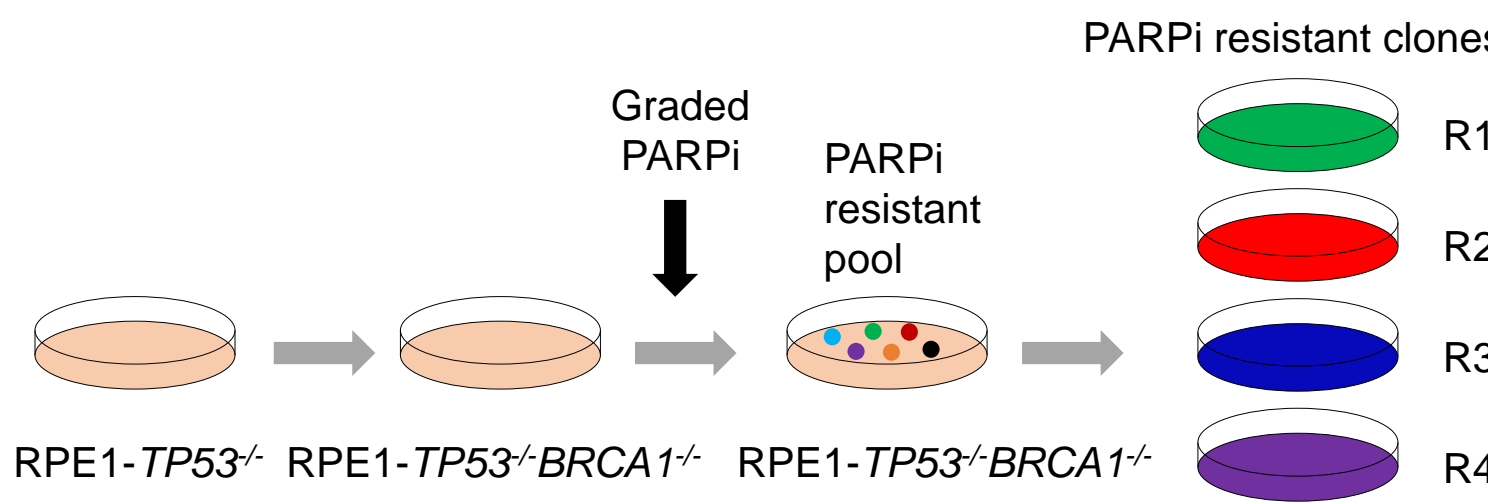

B

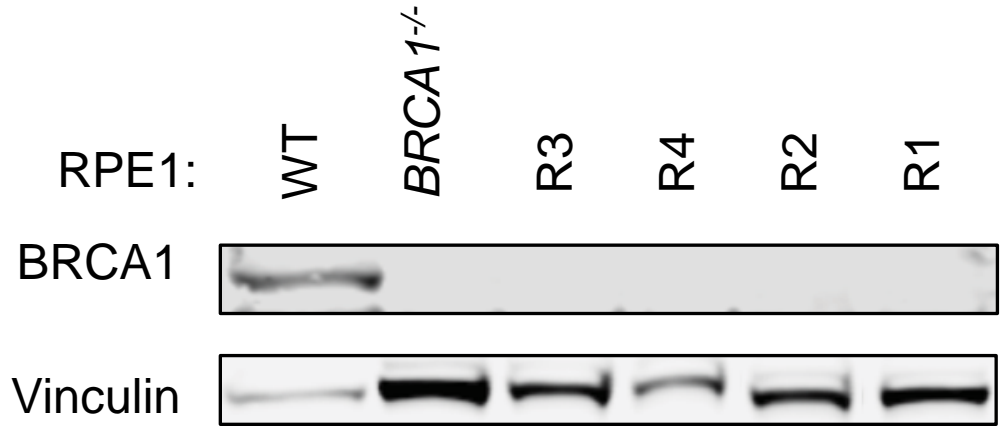

C

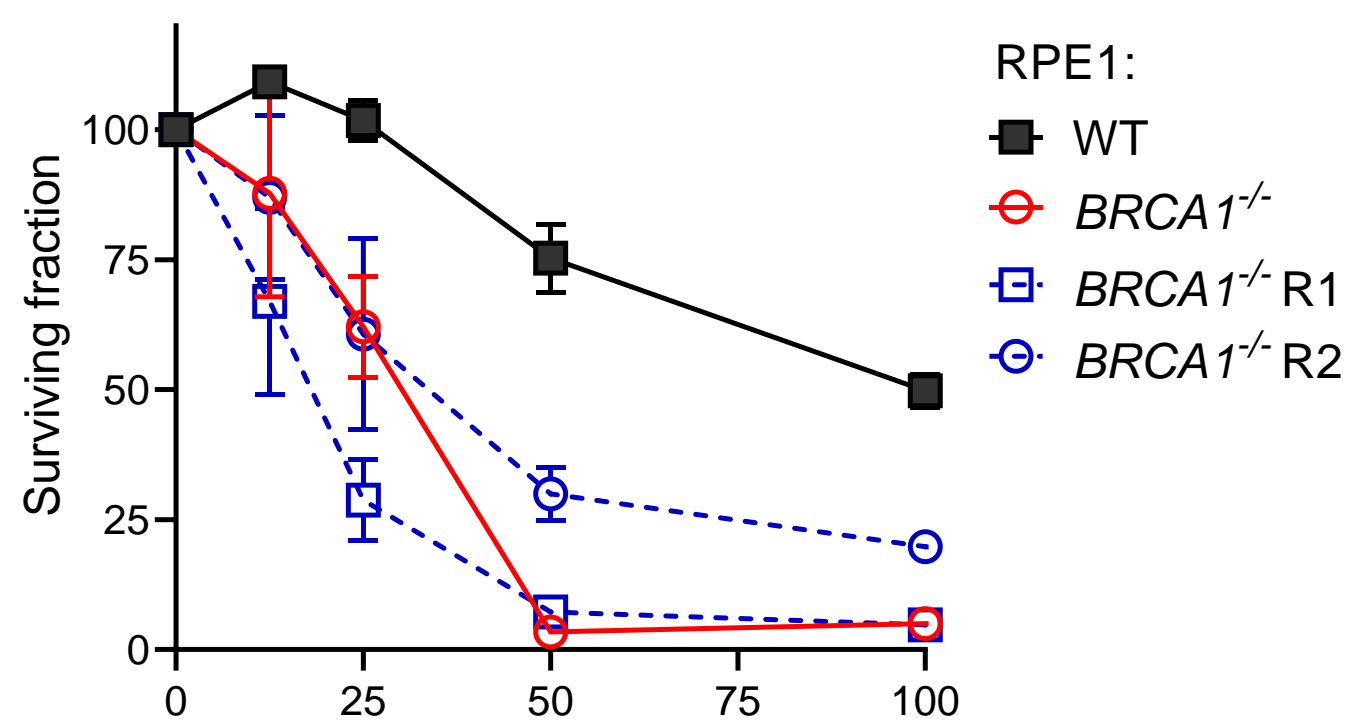

was preprint doi: https://doi.org/10.1101/2229.9523.11 1958; this version posted May 26, 2020. The copyright holder for this preprint (which

E
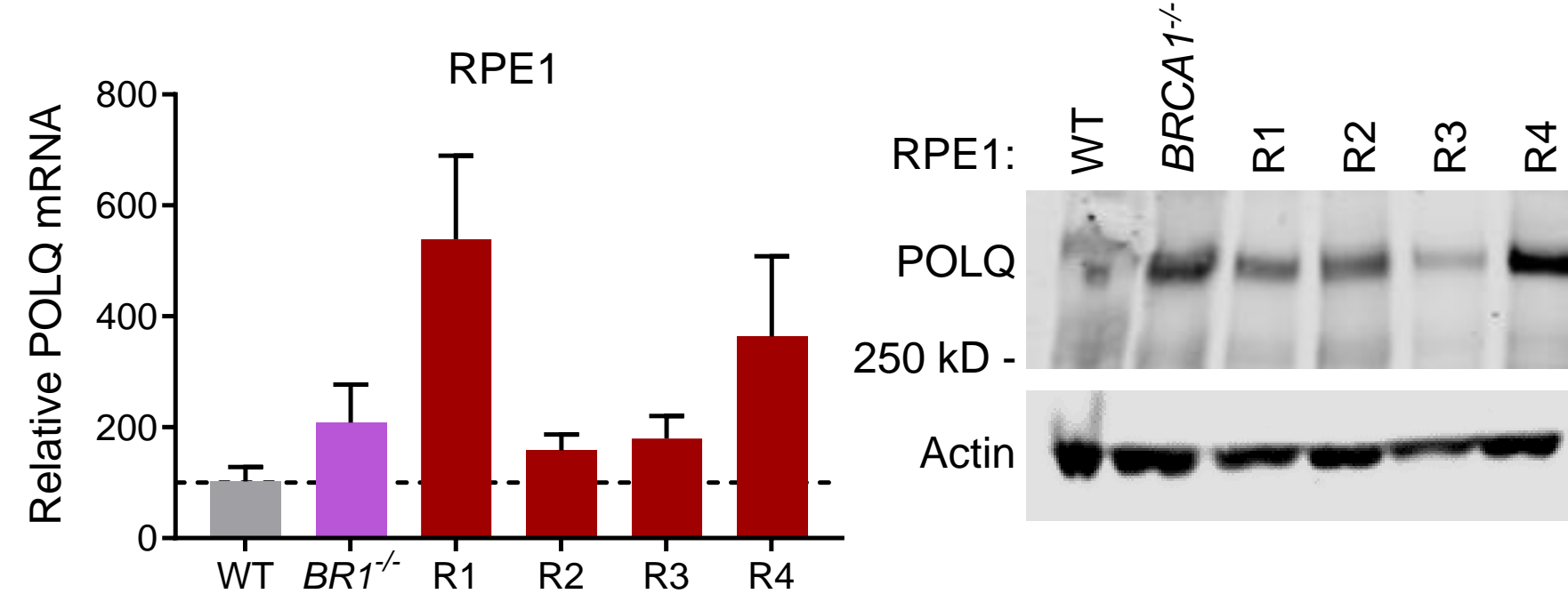

NVB sensitivity: $R \quad S \quad S \quad S \quad S \quad S$

$\begin{array}{lllllll}\text { PARPi sensitivity: } & R & S & R & R & R & R\end{array}$

G

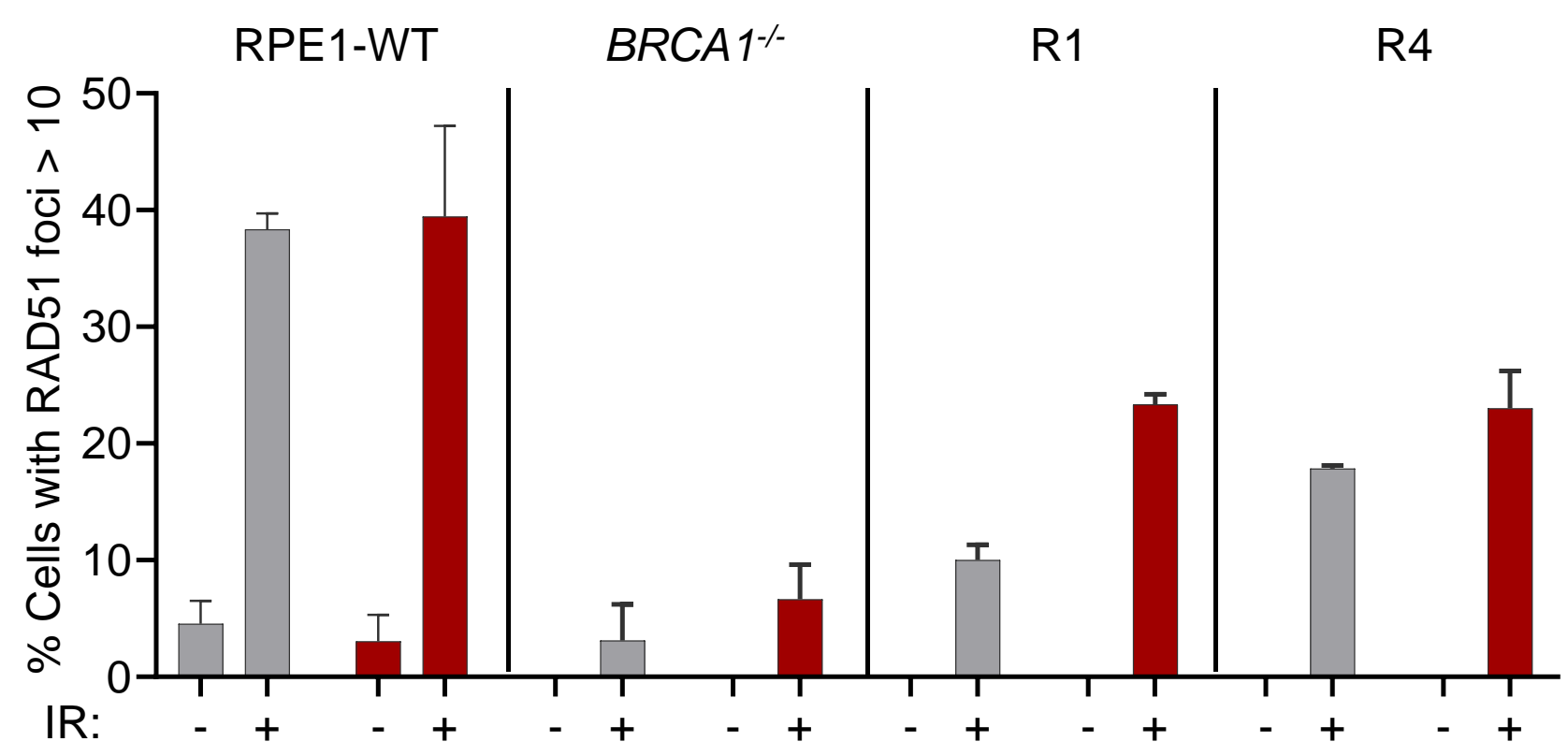

NVB:

I

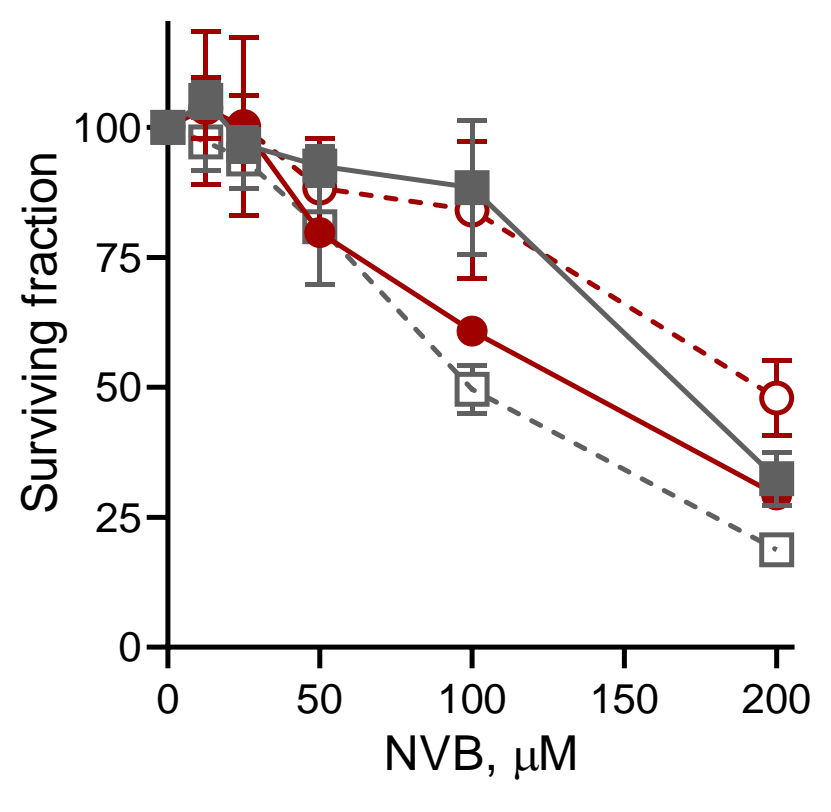

RPE1 siCtrl

- RPE1 SiRAD51

- R1 siCtrl RPE1: WT R1

o. R1 siRAD51

SiRAD51: -+-+
RAD51

Vinculin $\sim \sim-\sim$

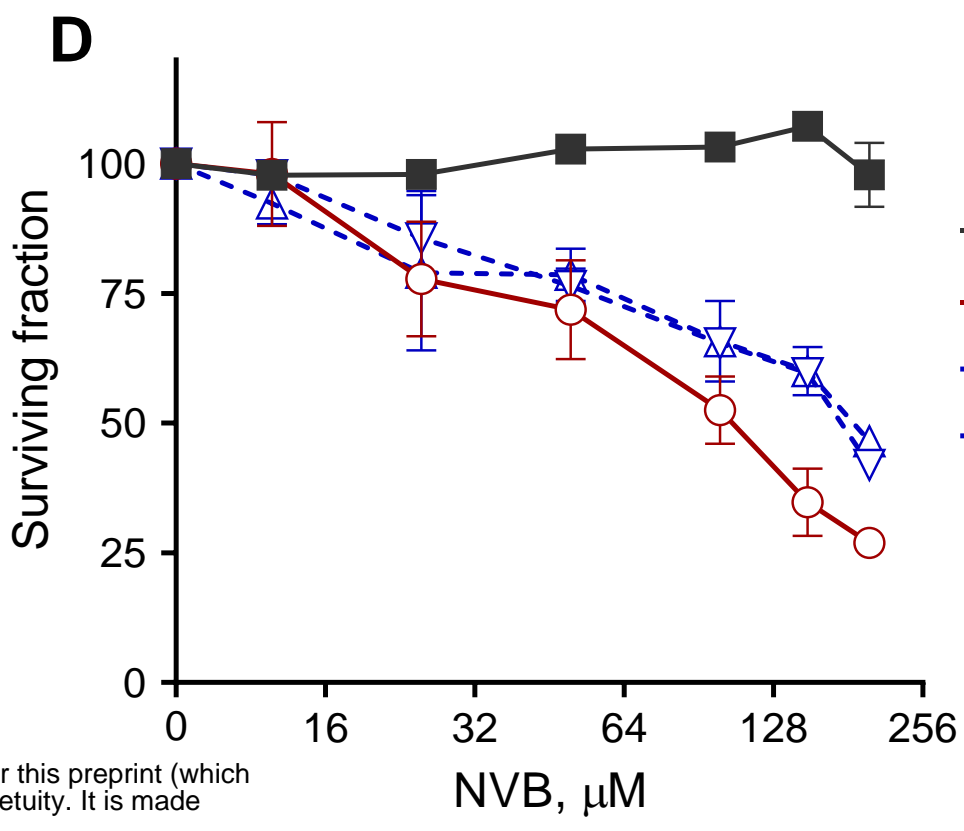

RPE1:

- WT

- $\mathrm{B} B R C A 1^{-/}$

$-\nabla \cdot B R C A 1^{-/-} \mathrm{R} 3$

$-A \cdot B R C A 1^{-/-} \mathrm{R} 4$
F

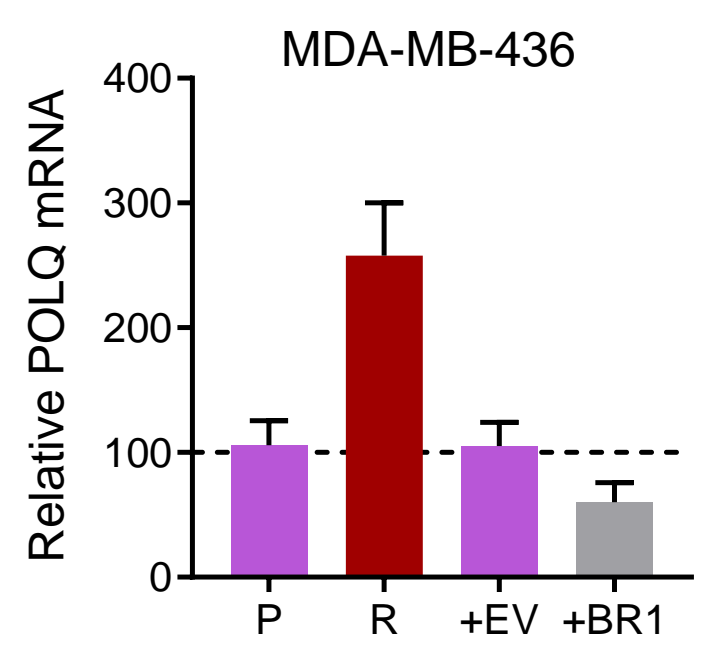

NVB sensitivity: $S \quad S \quad S \quad R$ PARPi sensitivity: $S \quad R \quad S \quad R$

H

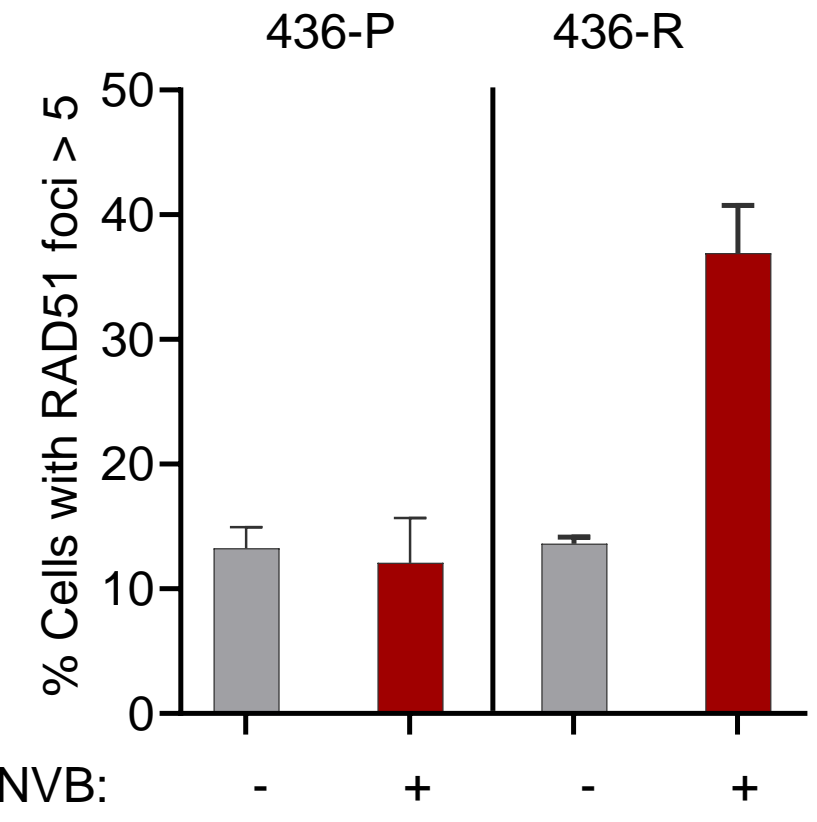

